\title{
Applications of Microwave Materials: A Review
}

\author{
ATHIRA RAVEENDRAN,${ }^{1}$ MAILADIL THOMAS SEBASTIAN,${ }^{2}$ \\ and SUJITH RAMAN $\mathbb{D}^{1,3}$
}

1.-Department of Electronics and Instrumentation, Bharathiar University, Coimbatore 641046, India. 2.-CSIR - National Institute for Interdisciplinary Science and Technology (NIIST), Trivandrum 695019, India. 3.—e-mail: sujithrpkd@gmail.com

The performance of microwave devices mainly depends on the properties of materials used in the fabrication. Knowledge of material properties at microwave frequencies is a prerequisite to select suitable materials for various microwave applications and vice versa. In this review, seven categories of materials and their applications in a microwave regime are elaborately discussed. The categories include magnetic materials, carbon-based materials, flexible or stretchable materials, biomaterials, phantoms, tunable materials and metamaterials. A brief overview of other important microwave materials such as low-loss ceramic dielectric materials, low-loss polymer ceramic composites, glass ceramics and multilayer ceramics is also given. The objective of this review is to expose the world of materials for wide microwave applications and thereby properly assisting the material selection for specific applications. Moreover, this review has dual significance. It helps material scientists to develop new materials and modify the properties of the available materials with respect to the application requirements. It also assists microwave engineers to select and use appropriate materials for different microwave applications.

Key words: Antennas, biomaterials, dielectric, materials, microwaves

\section{INTRODUCTION}

Humans started exploring new materials for daily pursuits and to attain a comfortable life during the Stone Age, followed by invention of copper and continued exploration for new materials to further improve quality of life. Hence, the investigation of new materials and their characterization have a major role in any field of science. Materials and microwaves are intertwined fields of research in the recent era of science and technology. The materials used in microwave frequencies $(300 \mathrm{MHz}-300 \mathrm{GHz})$ are termed as microwave materials. The propagation of electromagnetic waves in a material is characterized by the properties of that material. The wavelength and velocity of electromagnetic waves vary within a material medium according to

(Received August 7, 2018; accepted February 6, 2019; published online February 25, 2019) their dielectric properties. Microwave materials can be classified based on the unique properties exhibited by them and/or the chemical or physical structure of the material. The key parameters determining the properties of a material at microwave frequencies are complex permittivity and complex permeability. The selection of appropriate material for specific application mainly depends on the electrical and/or magnetic properties exhibited by the material and its inherent losses. The dielectric and magnetic losses are generally represented by loss tangents and are a function of operating frequency. Hence, material characterization at microwave frequencies is a prerequisite to select suitable materials for specific application. Application specific factors such as mechanical strength, weight, cost, availability and chemical and thermal stability of the material are also important in the material selection. For example, the microwave materials used for aerospace applications should have exceptional mechanical strength, light weight, 
conformity over surfaces, and chemical and thermal stability in addition to the electromagnetic performance of the device. Sebastian et al. have reviewed microwave materials for space applications. ${ }^{1}$ The major material requirement in aerospace and military applications includes electromagnetic shielding materials and the materials for high speed signal transmission. All these materials should be strong enough to withstand harsh environmental conditions. Since it is difficult to find a single material which exhibits all of these properties, various composites viz. low-loss dielectric ceramics, low-loss polymer ceramic composites, dielectric resonators, and multilayer ceramics are studied and synthesized for specific uses. These materials have been extensively studied for diverse microwave applications, and a large number of research articles have been published in this field. ${ }^{2,3}$ The advancement in this field has happened with the extensive support from other fields of science and technology such as nanotechnology, polymer science and material chemistry, which has resulted in smart and compact present-day devices.

Advanced material research has facilitated the replacement of bulky, expensive and poorly integratable traditional microwave devices with compact integrating devices. The constant demand for miniaturization of wireless communication devices and systems has promoted the research for lightweight and low-loss microwave materials with low sintering temperature. Microwave dielectric oxide ceramics play a key role in the development of lightweight circuit components for microwave and millimeter-wave frequencies. ${ }^{2}$ Since it is difficult to obtain an appropriate loss factor, optimum dielectric constant, good electrical and thermal properties in a single material, polymer composites are developed to combine the benefits of ceramics and polymers. In antenna applications, materials are mainly used in two ways: for fabricating the conductive part and the supporting substrate materials. Materials having tunable permittivity or permeability such as garnets, ferrites and liquid crystals can be employed in designing reconfigurable antennas. Composites and ceramics are generally recommended to use as substrates for achieving antenna miniaturization. Antennas designed over flexible substrates possess high mechanical flexibility which makes them suitable for wearable biomedical, military and space applications.

The discovery of carbon nanotubes (CNTs) and graphene accelerated the development of lightweight, compact, high-speed devices due to their inherent highest carrier mobility, and excellent electrical and thermal conductivity. The processing of graphene is more economical in comparison to CNTs. Active research is occurring all over the world to enhance the usage of these materials at microwave- and millimeter-wave frequencies. Nonetheless, recent developments in technology have realized antennas using graphene and CNTs. ${ }^{4,5}$ More advancements and research are required to improve their lesser radiation efficiency. Conventionally, metals are used for electromagnetic shielding due to their good electrical and thermal conductivity. But the corrosive nature of metals limits their applications. Later on, conductive polymers and carbon-based nanocomposites were proposed as lightweight shielding materials. ${ }^{6}$ Microwave-absorbing materials with large dielectric or magnetic losses have numerous applications in electromagnetic shielding, healthcare, stealth technology, etc. Carbonaceous materials were reported to be used as efficient fillers in polymer-based composite materials to improve microwave absorption characteristics. ${ }^{7}$ Meng et al. reviewed the recent progress in the development of graphenebased composites and their microwave absorption properties. ${ }^{8}$ A conductive concrete material with superior shielding effectiveness has been developed as an economical material for designing pyramidal absorbers in anechoic chambers by incorporating carbon black, graphite and steel fibers in polymer matrix. ${ }^{9}$

The anisotropic nature of ferrites has been utilized in designing non-reciprocal devices from the early days of microwave device fabrication. Later on, the requirement of more compact and highperformance microwave devices has led to the synthesis and characterization of magnetic nanocomposites, ferromagnetic thin films and ferromagnetic microwires. Ferromagnetic nanowirebased metamaterials are reported to have potential application in realizing spintronic devices at higher frequencies. ${ }^{10}$ Flexible electronics enable the integration of high-speed devices on flexible substrates which can be conformed over the human body or parts of automobiles and aircrafts. Jung et al. reviewed the development of different active and passive microwave devices designed on flexible or stretchable substrates. ${ }^{11}$ Among the ever-increasing use of nonbiodegradable materials in electronic industry, the work of Guna et al. has a great importance in the field of green electronics. They developed a completely biodegradable printed circuit board using biocomposites extracted from agrowastes. $^{12}$ The extensive use of wireless devices caused severe radiation hazards. Hence, the performance evaluation (e.g., radiation effect) of microwave devices is mandatory to ensure the quality of service. Phantom materials are proposed for this particular application for studying the interaction of electromagnetic waves with living tissues.

Even though some reviews are available in literature dealing with the microwave applications of a specific class of materials or materials used for specific microwave applications, there is no extensive review available in literature that gives a comprehensive discussion on microwave materials of various classes and their respective applications and vice versa (to the best of our knowledge). For 
example, Ahmed et al. reviewed the properties and applications of major electrically tunable materials at microwave frequencies, ${ }^{13}$ whereas Geetha et al. investigated different materials for electromagnetic shielding applications. ${ }^{14}$ In this work, we have tried to summarize different categories of materials and their respective applications at microwave frequencies. This review paper has dual significance in the present scenario. It paves a path for new researchers to understand the possible applications of various microwave materials and to explore their diverse applications. Thus, it helps in selecting appropriate materials for a particular application. Further, the classification of different categories of materials based on their microwave application assists active researchers in finding the field which needs more attention and research improvements. In this work, we review mainly seven categories of microwave materials based on their application.

1. Magnetic materials

2. Carbon-based materials

3. Flexible or stretchable materials

4. Biomaterials

5. Electromagnetic phantom materials

6. Tunable materials

7. Metamaterials

8. Other important microwave materials

- Low-loss ceramic dielectric materials

- Low-loss polymer ceramic composites

- Glass ceramics

- Multilayer ceramics

A brief overview of other important classes of microwave materials such as low-loss ceramic dielectric materials, low-loss polymer ceramic composites, glass ceramics and multilayer ceramics is presented in the "Other Important Materials" section. Some excellent publications are available in literature on microwave dielectric materials, ceramics and composites. ${ }^{2,3}$

\section{MICROWAVE MAGNETIC MATERIALS}

Magnetic materials have been used in microwave devices since the beginning of the microwave communication era. These materials are classified into different categories based on the alignment of magnetic dipoles in the presence of an external magnetic field as: ferromagnetic, anti-ferromagnetic and ferrimagnetic materials. They are mainly characterized by microwave permeability, and are widely used in microwave absorbers, tunable filters, antenna substrates and nonreciprocal passive devices. The current applications demand highspeed, efficient, compact and easily integratable microwave devices. This has lead to the use of ferromagnetic thin films, ${ }^{15}$ ferromagnetic microwires, magnetic nanocomposites, multiferroic materials and artificial magnetic materials. Some of the important microwave magnetic materials are listed below.

- Garnets

- Spinel ferrites

- Hexagonal ferrites

- Multiferroic materials

- Magneto-dielectric nanocomposites

- High-permittivity ferromagnetic materials

Ferrites have a long history as microwave material as they possess low eddy current loss, high permeability and moderate permittivity over a wide frequency band. ${ }^{16}$ Due to the nonreciprocal nature of these materials, they are used in the fabrication of microwave circulators and isolators. ${ }^{17}$ Ferrites are iron oxides containing divalent or trivalent metal ions $\left(\mathrm{Ni}^{+2}, \mathrm{Cu}^{+2}, \mathrm{Co}^{+2}, \mathrm{Mn}^{+3}, \mathrm{Y}^{+3}, \mathrm{Nd}^{+3}\right.$, etc.). Based on the arrangement of these atoms in the unit cell, ferrites are of three types: spinel ferrites, garnets and hexagonal ferrites. Among these ferrites, garnets exhibit excellent chemical and structural stability. The widely used yttrium iron garnets (YIGs) have narrow ferromagnetic resonance (FMR) and smaller magnetic loss. In the vicinity of FMR, permeability can be tuned significantly with a slight variation in the external biasing field. This property of YIGs has been utilized in the design of microwave oscillators ${ }^{18}$ and phase shifters ${ }^{19}$ with low insertion loss. Due to the high magnetic losses and resistivity, spinel ferrites have been investigated for microwave absorption properties. Xie et al. designed a thin wideband microwave absorber using NiCoZn spinel ferrites. ${ }^{20}$ Inverse spinel ferrites are also used in the development of microwave absorbers due to their inherent large magnetic losses. A flexible microwave absorber synthesized by dispersing an inverse spinel ferrite $\left(\mathrm{Ni}_{0.5} \mathrm{Zn}_{0.5} \mathrm{Fe}_{2} \mathrm{O}_{4}\right)$ in rubber matrix has been reported for stealth applications. ${ }^{21}$ Some analytical models are used to find suitable materials and their optimal composition for synthesizing magnetic composites with high microwave-absorption characteristics. ${ }^{22}$ Nickel ferrites, calcium ferrites and nickel-zinc ferrites are some of the magnetic composites used in the mitigation of electromagnetic pollution. ${ }^{22}$

At higher frequencies, electromagnetic waves cannot penetrate deep inside conductors due to the skin effect, so materials are used in the form of microwires, thin films, powder and flakes to increase the specific surface area for electromagnetic interaction. Setter et al. have reviewed the properties and diverse applications of ferromagnetic thin films. ${ }^{23}$ Soft magnetic materials have higher permeability and smaller hysteresis loss. Qiu et al. studied the soft magnetic properties of $\mathrm{FeCoB}$ and $\mathrm{FeCoNiB}$ thin films and proposed a microwave noise suppressor ${ }^{24}$ consisting of a bilayer of $\mathrm{FeCoB}$ and $\mathrm{SiO}_{2}$ thin films on a 


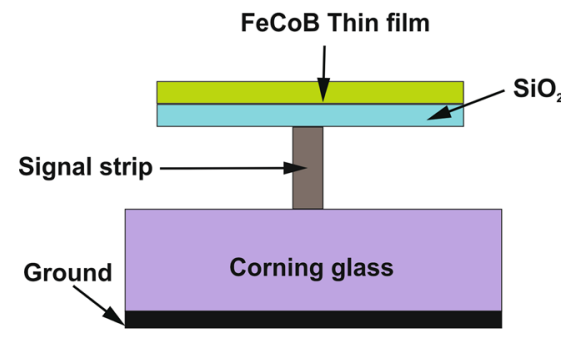

(a)

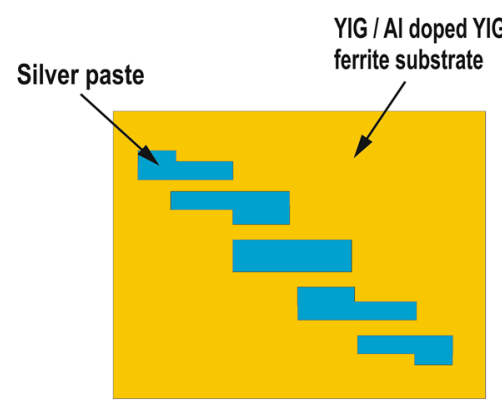

(c)

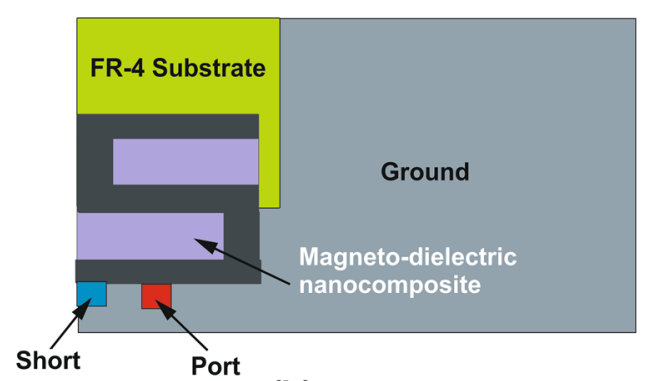

(b)

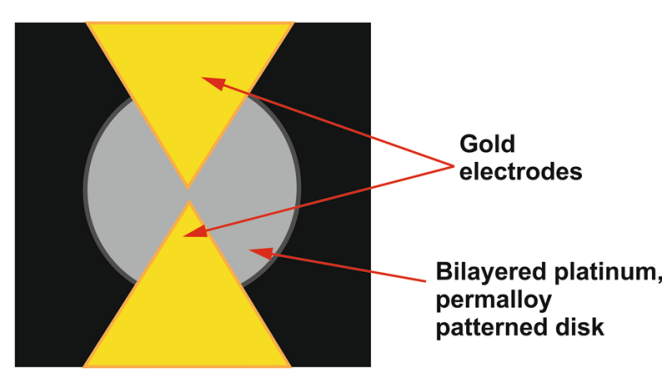

(d)

Fig. 1. Microwave devices using magnetic materials. (a) Magnetic thin film-based microwave noise suppressor, ${ }^{24}$ (b) Meander planar inverted-F antenna (PIFA) on magneto-dielectric nanocomposite (MDNC) material, ${ }^{34}$ (c) magnetically tunable band-pass filters ${ }^{37}$ and (d) spintronic device as nanoscale microwave oscillator ${ }^{15}$ (modified and redrawn using the data from Refs. 24, 34, 37 and 15).

microstrip line as shown in Fig. 1a. The ferromagnetic thin films greatly attenuate the transmission of a signal, which makes them promising for electromagnetic shielding applications. ${ }^{24}$ Certain magnetic composites such as composites filled with glasscoated ferromagnetic wires are reported as potential candidates for electromagnetic shielding applications due to their higher permeability and magnetic losses. ${ }^{25}$ Multiferroic materials are a unique class of materials exhibiting ferromagnetism, ferroelectricity and/or piezoelectricity, and their properties vary with the change in external magnetic field as well as electric field. They find application in antenna downsizing, tunable microwave devices and planar inductors. Makarova et al. proposed new multiferroic composites synthesized using NdFeb and lead zirconate titanate-26 (PZT-26) powder as fillers in polymer-based elastic matrix. ${ }^{26}$ Nowadays, magnetic materials are artificially engineered to achieve higher permeability and other desired electromagnetic properties. $^{27}$

\section{Applications}

\section{Electromagnetic Compatibility}

Electromagnetic interference (EMI) is one of the major issues affecting the performance of wireless systems. Hence, the development of electromagnetic shielding materials with exceptional shielding effectiveness has an ever increasing demand in all electronic systems. The attenuation of electromagnetic waves is mainly contributed by three mechanisms viz. absorption, reflection and multiple internal reflections. Qin et al. investigated the electromagnetic shielding effectiveness of ferromagnetic microwires embedded polymer composite. ${ }^{28}$ It has been found that thin layer of the proposed composite shows excellent shielding effectiveness compared to other conventional materials. The properties of a laminated shield fabricated using two copper layers and a CoNbZr layer have been investigated in Ref. 29. The proposed laminate exhibits higher shielding performance than conventional thick metallic sheet in ultra-high-frequency (UHF) bands. The laminated shield of high permeability shows large attenuation due to multiple reflections at conductive and magnetic layers. Therefore, its shielding performance is enhanced at FMR frequency.

\section{Antenna Applications}

YIG has been reported to be used as substrate in reconfigurable patch antennas due to the change in permeability of the material with the applied magnetic field. ${ }^{30}$ On applying a suitable magnetic field, the linearly polarized single-band antenna shows dual-band characteristics, and it shows circular polarization at two resonant frequencies. $\mathrm{Co}_{2} \mathrm{Z}$ hexaferrite is used as substrate in a compact tunable microstrip antennas in order to achieve significantly higher bandwidth and tunability. ${ }^{31}$ It has a low loss tangent and nearly equal permittivity and permeability. The greater tunability was achieved by the application of direct current (DC) magnetic bias along the width of the patch antenna. 
Guen et al. found the possibility of using porous ferrite ceramics as substrate instead of bulk materials for antenna miniaturisation. ${ }^{32}$ The dielectric and magnetic losses of the proposed porous ceramics were found to be strongly dependent on the temperature applied during the synthesis of the material. ${ }^{32}$ Ferrimagnetic YIG compound could be used as antenna substrate to suppress the mutual coupling between radiating elements in a multi-antenna system. It has been found that the direction of the applied external magnetic field plays a significant role in the reduction of mutual coupling. ${ }^{33}$ Han et al. demonstrated that the downsizing of an antenna can be achieved by using magneto-dielectric nanocomposite (MDNC) materials as substrate. ${ }^{34}$ The proposed substrate was synthesized by mixing cobalt nanoparticles in fluoropolymer matrix. A Meander planar inverted-F antenna (PIFA) designed based on MDNC material is depicted in Fig. 1b, and it exhibits acceptable performance with a low specific absorption rate (SAR) value. The high magnetic loss of composite material causes a reduction in back-side radiation. It is reported that magnetic FeCo nanoparticles or ferroelectric thin films can be used as substrate in stacked antennas for enhanced gain and tunability at WiFi bands. ${ }^{35}$ On the variation of external magnetic field, good improvement in the tunable frequency range was observed. Multiferroic magneto-dielectric materials are reported to be used as substrate in phase array antennas. ${ }^{36}$ It facilitates the development of a low-profile planar antenna whose radiation pattern can be configured by applying suitable voltage across the structure. The proposed multi-layered multiferroic substrate is composed of lead zirconate titanate, YIG and gadolinium gallium garnet. ${ }^{36}$

\section{Planar Microwave Devices}

Zhang et al. proposed a magnetically tunable microstrip band-pass filter using YIG and aluminum-doped YIG substrate in Ref. 37. The schematic of the prototype is as shown in Fig. 1c. The bandpass filter exhibits low insertion loss and the tunability is achieved by varying the external magnetic field. Inductors with a high quality factor, high inductance and low eddy current loss are developed using $\mathrm{FeGaB} / \mathrm{Al}_{2} \mathrm{O}_{3}$ multilayers, and they can be easily integrated in radio-frequency (RF) circuits. ${ }^{38} \mathrm{~A}$ disc of ferrite low-temperature cofired ceramic (LTCC) material has been used in a compact edge-guided circulator, unlike the traditional circulators using ferrite material surrounded by dielectric material. ${ }^{39}$ The proposed circulators can be easily integrated with other RF components in system-on-package with good isolation. Further, Yang et al. extended their studies by integrating windings within the ferrite material in order to reduce demagnetization. ${ }^{40}$ Then, the device performed multifunctionally; with bias current, it operated as a circulator, and without bias, it worked as a power splitter. A circulator's operating frequency can be adjusted by controlling the current through the windings in the presence of an external magnetic field. Lin et al. investigated the properties of multiferroic materials to control electric polarization by an external magnetic field or variation of magnetization with applied electric field. Based on this concept, they designed lightweight and powerefficient voltage or magnetic field-tunable microwave devices and magneto-electric sensors. ${ }^{41}$

\section{Waveguide Devices}

Magnetic opal nanocomposites are used to model circulators in microwave communication circuit systems. ${ }^{42}$ S-parameters of magnetic opal-based circulators are measured using the wavequide technique. The data obtained from numerical analysis shows good agreement with the experimental results. The modelled circulator shows good isolation, smaller insertion loss and larger bandwidth in comparison to ferrite-based conventional circulators. ${ }^{42}$ A surface-integrated waveguide-based phase shifter with bias windings is fabricated using multilayer ferrite LTCC material. ${ }^{43}$ Under symmetrical bias, it gives a reciprocal phase shift, whereas in anti-symmetrical bias, the phase shift is nonreciprocal. The proposed phase shifter doesn't require any external magnetic field for biasing, which allows large size reduction and facilitates integration in mobile phased arrays.

\section{High-Quality Transmission Lines}

Conductor loss degrades the performance of highspeed electronic systems at higher frequencies due to the skin effect. Therefore, high-quality transmission lines with reduced ohmic loss are necessary for microwave circuits operating in the frequency range from $12 \mathrm{GHz}$ to $40 \mathrm{GHz}$. Rahmi et al. investigated a multilayered transmission line of $\mathrm{Cu} / \mathrm{Ni}$ pairs to minimize the skin effect. ${ }^{44}$ The ferromagnetic material $(\mathrm{Ni})$ has negative permeability and produces reverse eddy currents which cancel out positive eddy currents produced by copper. The eddy current cancellation results in skin effect mitigation and reduction of ohmic loss.

\section{Spintronic Devices}

Spintronics or spin-based electronics use the spin of electrons to handle data and thereby enable highspeed communication, high-density packing of circuit components and development of nonvolatile storage devices. It is promising technology in the field of microwave communication to develop nanoscale oscillators and microwave detectors. A magnetic nanoscale spintronic device reported in Ref. 15 operates as a tunable microwave source. The device consists of a platinum and permalloy thin films with sharp-pointed gold electrodes as shown in Fig. 1d. 
The voltage applied between the electrodes induces pure spin current which in turn results in the spin Hall effect. Since the oscillation frequency varies with the strength of the magnetic field, it could be used as a tunable microwave source in future nanoscale microwave oscillators. The multilayer tunable spintronics device proposed by Chen et al. doesn't require bulky electromagnets to control magnetic resonance as in the conventional spintronic devices. ${ }^{45}$ The magnetic resonance in the CoFe-based spintronic device can be tuned by changing the interlayer coupling with a variable electric field. Hence, it allows high-density integration of devices with low power consumption and fast response.

\section{Biomedical Applications}

Microwave hyperthermia is an effective method for cancer treatment in which tumor cells are heated locally. Biocompatible magnetic nanoparticles such as magnetite $\left(\mathrm{Fe}_{3} \mathrm{O}_{4}\right)$ coated with polyacrylic acid are reported to be used in hyperthermia. ${ }^{46}$ Due to high magnetic loss associated with the magnetic nanoparticles (MNPs), the specific absorption rate increases, which in turn increase generation of heat. The temperature rise in the biological tissues injected with MNPs is high compared to normal tissues without MNPs. Thus, this noninvasive method provides increased heating efficiency with good material selectivity and low power requirement $(<1 \mathrm{~W})$.

\section{CARBON-BASED MATERIALS}

Carbon is popularly known as the building block of living things, which are mainly used for absorption applications in the microwave regime. Diamond, graphite, carbon black, CNTs and graphene are some of the allotropes of carbon which find numerous applications in microwave electronics. The discovery of CNTs and graphene created a tremendous development in the field of high-speed microwave electronics as they possess high carrier mobility and high electrical and thermal conductivity. ${ }^{47}$ They are recommended as an excellent alternative for traditional silicon-based integrating circuits. They also possess high tensile strength and a large Young's modulus which makes them suitable for flexible electronics. ${ }^{47}$ The electrical properties of single-walled CNTs (SWCNTs) depend on the chirality of the structure. Graphene is a zeroband-gap material in natural form. One of the common methods to open the band gap is to chemically modify graphene to reduced graphene oxide (r-GO). CNT and r-GO-based diodes are reported as high-speed switches for millimeter-wave circuits. A schematic of the proposed diode is illustrated in Fig. 2a. Graphene has very high carrier mobility ( $>10^{5} \mathrm{~cm}^{2} / \mathrm{Vs}$ ), optical transmittivity and stability at room temperature. ${ }^{48}$ The graphene-based metal-oxide-semiconductor field- effect transistor (MOSFET) investigated in Ref. 48 shows excellent switching characteristics. The schematic of the proposed MOSFET is shown in Fig. 2b. In addition, the impedance of graphene can be easily controlled to match with other RF integrating circuit components in comparison to CNT-based RF devices. ${ }^{49}$ Generally, CNTs exhibit high impedance (20 $\mathrm{k} \Omega-10 \mathrm{M} \Omega$ ). Diamond is another potential candidate for high-frequency switching applications, as it possesses a large band gap, high breakdown electric field strength, high electron and hole mobility and thermal conductivity. ${ }^{50}$

It is difficult to achieve proper impedance matching in single-dielectric or magnetic lossy material, in which dielectric and magnetic materials are mixed together in a proper ratio. Khani et al. synthesized a heterostructure containing dielectric and magnetic parts; that is, iron nanoparticles coated with a lowdensity carbon shell which showed stable absorption properties. ${ }^{51}$ It has been reported that CNTs filled with magnetic particles ( $\mathrm{Fe}, \mathrm{Co}, \mathrm{Ni}),{ }^{52}$ CNT-filled ferromagnetic alloy nanowires, ${ }^{53}$ magnetic particle/carbon-based nanohybrids, ${ }^{54}$ hexagonal boron nitride nanocrystal (BNNC)/graphene nanoflakes composites $^{55}$ and carbon black/silicon carbide composites $^{56}$ exhibit excellent microwave absorption characteristics. Qin et al. have reviewed microwave absorption properties of different carbon-based polymer composites. ${ }^{7}$ Carbon-based polymer composites are lightweight absorbers which demonstrate higher microwave absorption and mechanical flexibility. Carbon black, carbon fibers, CNTs and graphene can be used as fillers in polymer matrix in order to increase conductivity. Microwave absorption characteristics vary with the composition, geometry and morphology of carbonaceous particles. $^{7}$ Sebastian et al. investigated microwave absorption properties of SWCNT-filled polytetrafluoroethylene (PTFE) composites. ${ }^{57}$ They found that the dielectric constant and power attenuation coefficient increase with SWCNT loading. The large number of free electrons present in the SWCNT are responsible for the increase in dielectric losses. ${ }^{57} \mathrm{~A}$ conductive concrete has been proposed as alternative for commercially available carbon-laced polyurethane absorbers used in microwave anechoic chambers. ${ }^{9}$ It has been prepared by the addition of carbonaceous materials such as carbon black, graphite powder, carbon fibers and steel fibers in concrete material. The dispersed carbonaceous particles cause multiple internal reflections and produce eddy currents within the concrete material which in turn increases the shielding effectiveness. They are very cost-effective and easily available.

The synthesis and extraction of pure carbon nanofibers (CNFs) is complex and costly. Joseph et al. synthesized a simple, lightweight and low-cost electromagnetic shielding material by incorporating graphite flakes in polyvinylidene fluoride (PVDF) ${ }^{58}$ The shielding mechanism is mainly due to absorption, and a material of 0.5-mm thickness shows EMI 


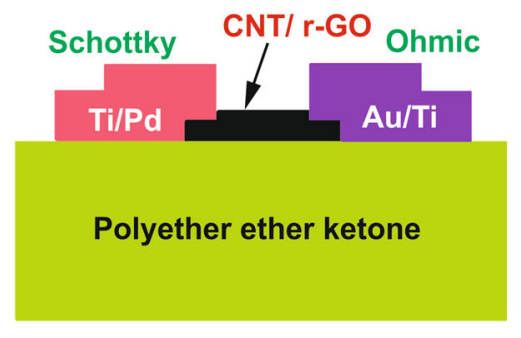

(a)

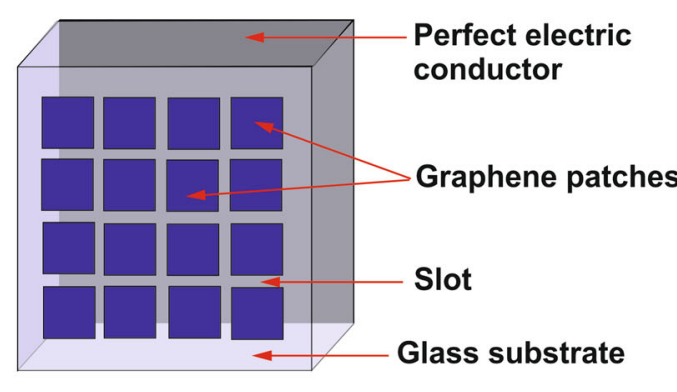

(c)

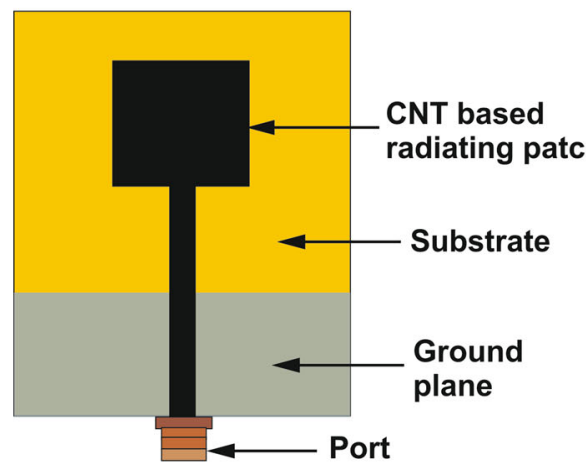

(e)

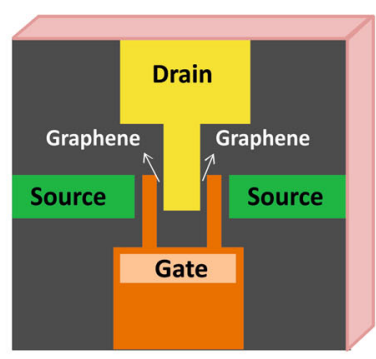

(b)

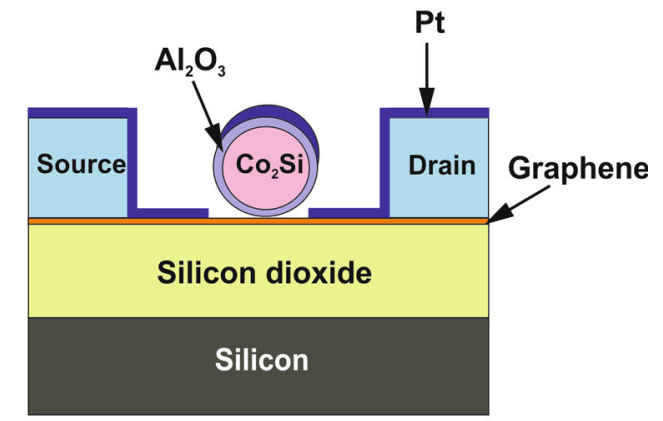

(d)

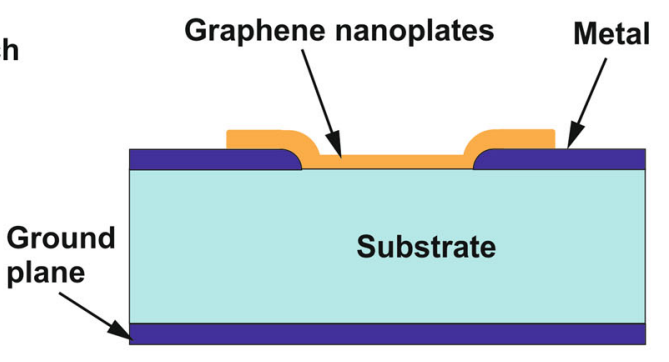

(f)

Fig. 2. Microwave devices using carbon-based materials: (a) CNT/r-GO-based diode (modified and redrawn using the data from Ref. 47), (b) graphene-based field effect transistor (modified and redrawn using the data from Ref. 48), (c) graphene-based tunable absorber (modified and redrawn using the data from Ref. 68), (d) graphene-based high-speed transistor adapted with permission from Ref. 71, copyrighted by Springer Nature, (e) CNT-based antenna (modified and redrawn using the data from Ref. 73) and (f) graphene-based microstrip attenuator (modified and redrawn using the data from Ref. 82).

shielding effectiveness (EMI SE) of more than 50 $\mathrm{dB}$. They continued the investigation on electromagnetic shielding materials and developed a flexible, low-cost electromagnetic shielding fabric. ${ }^{59} \mathrm{~A}$ composite of polyaniline nanofibers with graphite has been synthesized and its performance has been compared with polyaniline nanofibers. The variation in EMI SE of polyaniline nanofibers and graphene-filled polyaniline nanofibers with frequency is depicted in Fig. 3. The overall EMI SE is the combination of shielding effectiveness due to absorption $\left(\mathrm{SE}_{A}\right)$ and shielding effectiveness due to reflection $\left(\mathrm{SE}_{R}\right)$ mechanisms. The proposed graphene-filled polyaniline fabric of 1-mm thickness shows an overall shielding effectiveness of more than $80 \mathrm{~dB}$ over the frequency range $8.2-18 \mathrm{GHz}$. Later, polyaniline nanofiber/graphite compositebased cotton nylon fabrics were developed using in situ polymerization. The proposed fabric of thickness $0.1 \mathrm{~mm}$ showed more than $11 \mathrm{~dB}$ shielding effectiveness in the frequency range 8.2$18 \mathrm{GHz} .{ }^{59}$

Artificial tissue-mimicking materials are required to test the quality and safety of various biomedical diagnosis techniques. Carbon powders such as graphite and carbon black are added in tissuemimicking materials in order to control the conductivity and permittivity. Flexible and electrically stable phantoms were developed by mixing carbon powders with urethane rubber matrix. ${ }^{60}$ The 


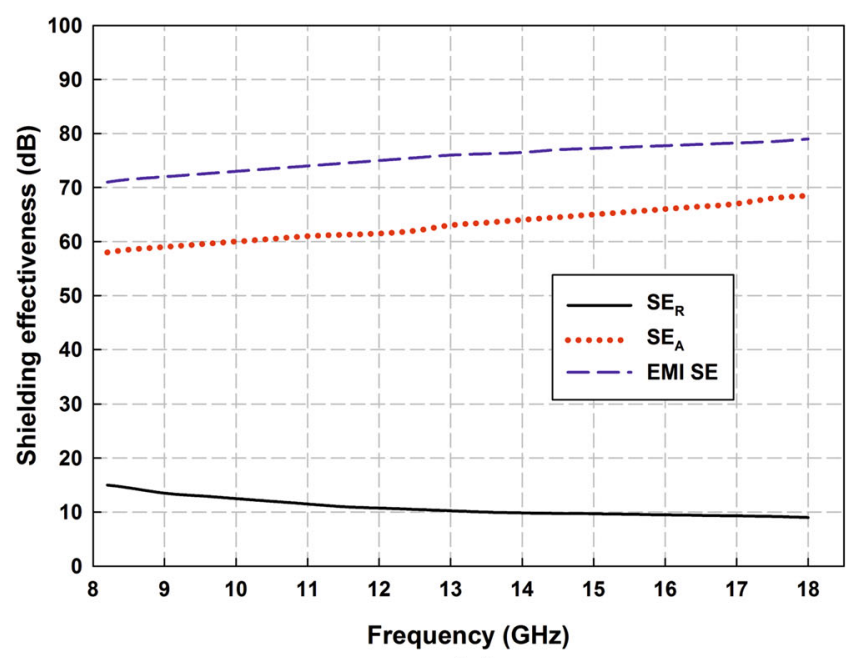

(a)

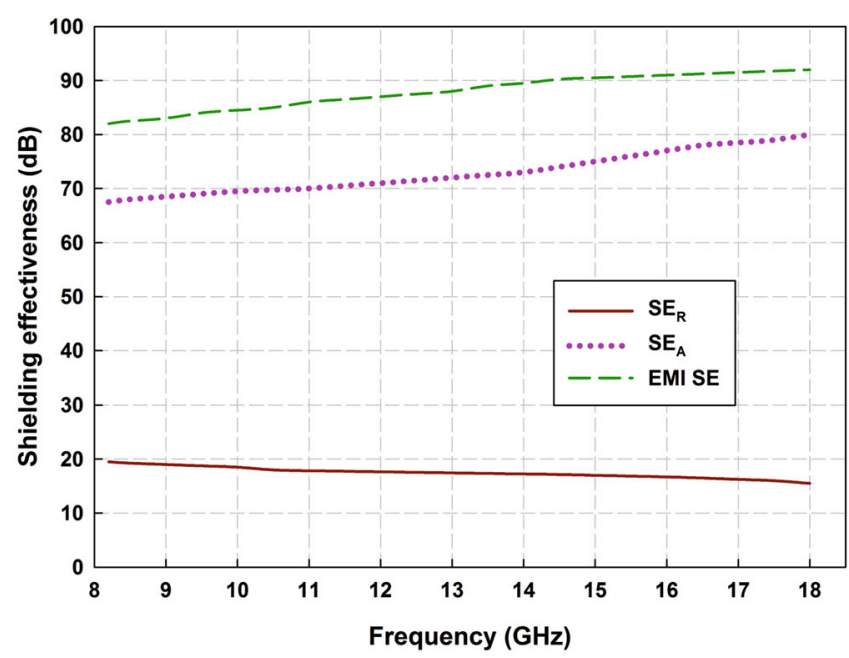

(b)

Fig. 3. Variation in shielding effectiveness in the frequency range $8.2-18 \mathrm{GHz}$ : (a) polyaniline nanofibers of thickness $1 \mathrm{~mm}$ and (b) graphenefilled polyaniline nanofibers of thickness $1 \mathrm{~mm}$ (redrawn using the data from Ref. 59).

permittivity and conductivity can be controlled by the concentration of carbon powders in order to mimic dielectric properties of human tissues. Some of the major applications of carbon-based materials in the microwave regime are discussed below.

\section{Applications}

\section{Electromagnetic Compatibility}

A thin film of CNTs can be used as radarabsorbing material. ${ }^{61}$ A composite material has been synthesized by mixing alumina nanoparticles with CNTs. ${ }^{61}$ As the concentration of CNTs increases, the conductivity of the material increases, which in turn decreases skin depth. Therefore, such composite material can be used as electromagnetic shielding material since skin depth is inversely proportional to wave attenuation. CNFor multiwalled CNT (MWCNT)-filled silicon rubber composites and CNF wafer-sandwiched silicone rubber composites have a lot of application in electromagnetic shielding. ${ }^{6}$ Among these carbonbased nanocomposites, CNF-sandwiched silicone rubber composites show superior shielding effectiveness and absorption. A flexible electromagnetic shielding material has been reported for industrial applications and it was developed by dispersing graphene on a web made up of stainless steel fibers. ${ }^{62}$ CNTs of low density and higher electrical and thermal conductivity are suitable for developing radar-absorbing materials (RAMs) and electromagnetic shielding materials. ${ }^{63}$ The EMI shielding increases with increase in the conductivity of CNTs.

\section{Microwave Absorbers}

MWCNTs are embedded with magnetic nanoparticles like $\mathrm{Fe}$, Co and $\mathrm{Ni}$ to enhance electromagnetic absorption in the S-band. ${ }^{52}$ Hence, they can be used to realize lightweight high-performance microwave absorbers. Helical CNF-coated carbon fiber is an efficient material for microwave absorbers due to its chemical stability, low density and broad absorption characteristics. ${ }^{64}$ Lightweight magnetic composites of glass-iron-carbon exhibit good microwave absorption over a wide frequency band. ${ }^{65}$ Hong et al. investigated microwave absorption properties of titanium carbonitride and found that thin microwave absorbers can be realized by optimal doping of carbon in the compound. ${ }^{66}$ Silica-nickel-carbon composites are also reported to have good absorption characteristics. ${ }^{67} \mathrm{~A}$ metasurface containing graphene patches as shown in Fig. 2c could be used as a tunable microwave absorber as the surface resistance of graphene varies with $\mathrm{DC}$ voltage or doping concentration. ${ }^{68}$ The impedance of the graphene-based metasurface can be varied by an external electric field which in turn controls resonant frequency. ${ }^{68}$

\section{RF Integrating Devices}

CNT-based field effect transistors (FETs) have been discussed in Ref. 69 and they contain a large number of CNTs in the channel region which allows flow of high current. A maximum stability gain of 10 $\mathrm{dB}$ is shown which make them suitable for highfrequency amplifiers and oscillator circuits. CNTbased FETs can be used in designing different logic gates like NOT, NAND and NOR. ${ }^{9}$ Graphene is suitable for the development of high-speed graphene-based FETs (GFETs) due to its inherent high carrier mobility. The geometry of a high-speed GFET is shown in Fig. 2d. A GFET with higher cut-off frequency has been modelled using $\mathrm{Co}_{2} \mathrm{Si}_{-}-\mathrm{Al}_{2} \mathrm{O}_{3}$ nanowires as gate material. ${ }^{71}$ Russell et al. studied the effect of varying the gate length in a diamond-based RF FET. ${ }^{50}$ They found that a 
higher cut-off frequency of $45 \mathrm{GHz}$ has been achieved for an RF FET developed on single diamond substrate with a gate length of $120 \mathrm{~nm}$. Aldrigo et al. proposed a tunable microwave filter using CNT-based varactor diodes. ${ }^{72}$ CNT-based varactor diodes are less lossy and have higher cutoff frequency compared to other semiconductorbased varactors. CNT- and r-GO-based diodes are promising candidates for microwave rectification. ${ }^{47}$ r-GO diodes also find application in frequency multipliers and mixers. ${ }^{47}$ They can be integrated into RF circuits since they exhibit optimal impedance and low parasitic effects.

\section{Antenna Applications}

A microstrip-fed monopole antenna has been developed using SWCNT composites. ${ }^{73}$ The geometry of the aforementioned antenna is shown in Fig. 2e. The radiating patch and ground plane is fabricated using CNT composites. The CNT composite-based antenna shows wider bandwidth compared to a conventional copper antenna operating in the same frequency range, but it has less efficiency. A graphene-based tunable slot antenna fabricated on $\mathrm{SiO}_{2}$ layer shows wider bandwidth compared to a metallic antenna of the same geometry working at the same frequencies. ${ }^{74}$ Graphene-based radiation reconfigurable antennas were also reported in literature. ${ }^{4}$ Graphene-based ink has been used to design and fabricate a printable dipole antenna for $\mathrm{RF}$ identification (RFID) tags at UHF bands. ${ }^{75}$ Even though it has some limitations in the interrogation range of tags due to the large resistance of the graphene sheet, it can be considered as an alternative for an expensive silver ink-based antenna. The serious issue associated with all these antennas is low radiation efficiency. Recently, an efficient antenna fabricated using CNT threads has been reported in Ref. 5. Bengio et al. have coined a term called specific radiation efficiency to express a compromise between weight and radiation efficiency of the antenna. ${ }^{5}$ The proposed antenna showed nearly 20 times more efficiency compared to a copper wire antenna. It finds applications in wearable electronics and aerospace industries due to mechanical robustness and flexibility. Silicon carbide and carbon-reinforced fiber sandwich structures can be used to make reflectors in an antenna due to high specific stiffness and a low coefficient of thermal expansion (CTE) ${ }^{76}$ High surface accuracy of the proposed reflectors enables designing of lightweight and high-gain reflector antenna systems for space applications.

\section{Biomedical Applications}

Oil in gelatine mixture doped with carbon materials is a potential material for the development of phantoms for microwave imaging applications. ${ }^{77}$ CNTs, graphene ink and lignin are used for doping. The dielectric constant and conductivity increases by the addition of more CNTs and graphene ink due to parasitic capacitance. The excess addition of lignin causes a slight decrease in dielectric constant and conductivity due to saturation. An ultra-wideband (UWB) antenna has been proposed as a sensor for microwave medical imaging systems to detect brain tumors. ${ }^{78}$ The radiating patch has been realized using graphene on a polymeric substrate made of $\mathrm{Fe}_{2} \mathrm{O}_{3}$ and polydimethylsiloxane (PDMS). The magneto-dielectric substrate allows reduction of the antenna size and UWB characteristics. The high conductivity of graphene and low dielectric constant of PDMS facilitate production of higher energy radiation for detection of tumors.

\section{Sensors}

An UWB RFID system has been developed to sense methane at room temperature. ${ }^{79}$ It consists of an antenna and sensor head of SWCNTs loaded with palladium. The interaction of methane with the sensor head causes current flow, and it varies according to the concentration of methane. The possibility of a capacitive sensor for detection of gases has been studied in Ref. 80. It consists of two band-pass resonators and a sensitive layer of polymer composite doped with MWCNTs.

\section{Waveguide Devices}

Different forms of carbon are reported to be used as an attenuator in travelling wave tube. ${ }^{81}$ Due to its lossy nature, it gives better operating stability by reducing reflections. Pierantoni et al. designed tunable attenuators using graphene flakes, ${ }^{82}$ and the geometry of the proposed attenuator is depicted in Fig. 2f. The tunability is based on the variation in the resistance of graphene with the applied DC voltage.

\section{Spintronic Devices}

The spin property of particles is used for information processing and data storage in spintronics. Peng et al. investigated the use of carbon-based materials for spintronics applications. ${ }^{83}$ They found that graphene, CNTs and fullerene are potential candidates for low-power and high-speed spintronic devices.

\section{FLEXIBLE AND STRETCHABLE MATERIALS}

Flexible and stretchable materials find numerous applications in the emerging wearable electronics and biomedical field. In general, flexible or stretchable materials are materials possessing flexibility and/or stretchability. ${ }^{11}$ They can be easily integrated into textile fabric or implanted in biological systems. This technology facilitates the integration of circuit components on thin lightweight substrate materials providing excellent flexibility and stretchability. Microwave wearable devices designed on flexible substrates show high data accuracy due to 


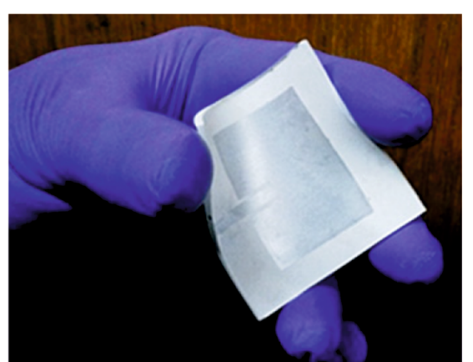

(a)

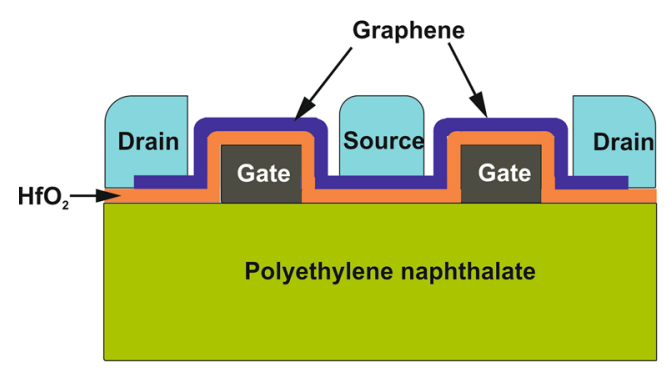

(c)

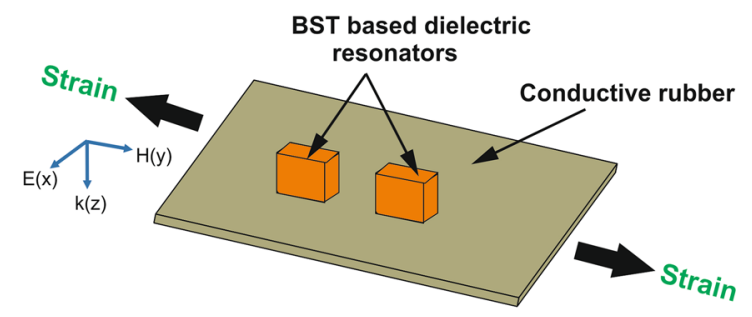

(e)

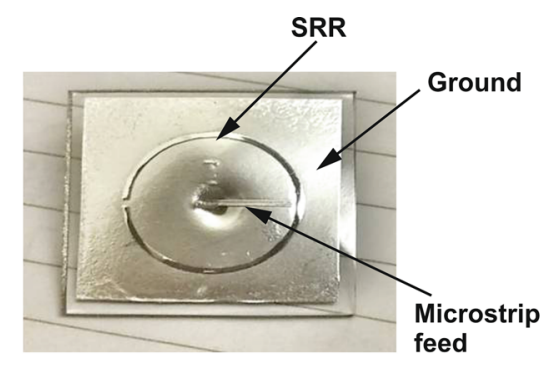

(b)

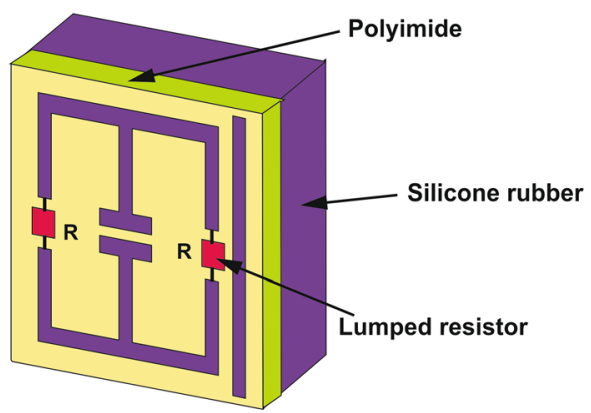

(d)

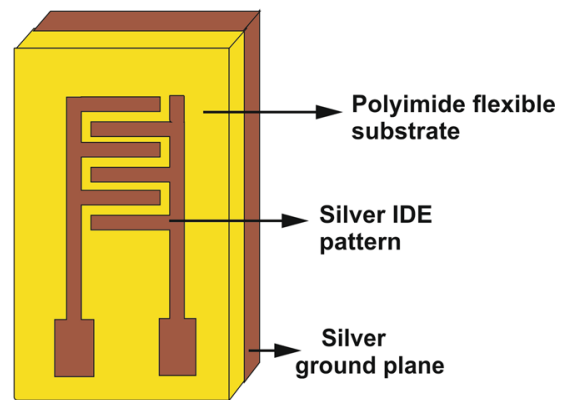

(f)

Fig. 4. Microwave devices using flexible materials: (a) photograph of flexible microstrip patch antenna using silicone rubber, (b) photograph of flexible antenna using PDMS substrate, (c) flexible GFET on PEN substrate (modified and redrawn using the data from Ref. 90), (d) flexible metamaterial-based absorber (modified and redrawn using the data from Ref. 99), (e) mechanically tunable absorber on conductive rubber adapted with permission from Ref. 102, copyrighted by AIP Publishing and (f) flexible sensor on polyimide adapted with permission from Ref. 119, copyrighted by Taylor \& Francis.

their conformity over the human body. A photograph of a flexible microstrip antenna fabricated on silicone rubber substrate is shown in Fig. 4a.

The active or passive microwave devices are composed of mainly two materials: conducting material and the supporting substrate. In literature, many flexible or stretchable substrate materials have been reported, such as liquid crystal polymer (LCP), ${ }^{84}$ polydimethylsiloxane (PDMS), ${ }^{85}$ silicone rubber, ${ }^{86}$ urethane rubber, ${ }^{86}$ polyethylene terephthalate (PET), ${ }^{87}$ polyvinylidene difluoride (PVDF) ${ }^{88}$ Kapton polyamide ${ }^{89}$ and polyethylene naphthalate (PEN). ${ }^{90}$ These materials are generally characterized by light weight, excellent mechanical flexibility or stretchability and low dielectric constant. LCP is a low-cost water-resistant packing material with low dielectric constant and low loss tangent. Its CTE can be controlled by proper heat treatments, and it facilitates vertical integration of RF components. ${ }^{84} \mathrm{LCP}$ is also a promising antenna substrate for flexible multilayer antenna array systems. ${ }^{84}$ PDMS is the most commonly used elastomeric fluid in flexible electronics and an example of a PDMS-based antenna is shown in Fig. 4b. PDMS has a low dielectric constant (2.77-3.69), excellent chemical stability and good optical transparency. ${ }^{85}$ It has been widely used in implantable biomedical devices due to its biocompatibility. ${ }^{91}$ At millimeter-wave frequencies, it exhibits a high loss tangent $(\approx 0.04)$ and a low Young's modulus $(<2 \mathrm{MPa}){ }^{91} \mathrm{SU}-8$ is a flexible polymer usually attached to PDMS substrate in flexible electronic systems as a photo-resist and it is comparatively easier to handle at wafer levels. ${ }^{92}$ Kapton polyamide exhibits low dielectric loss, greater thermal stability ${ }^{89}$ and an ultimate tensile strength of 
$231 \mathrm{MPa} .{ }^{85}$ Conventionally, thin copper foils are used as flexible conducting material, ${ }^{93}$ but they are not stretchable. Liquid conductors are good alternatives for obtaining excellent mechanical stretchability, bendability and twistability. Mercury is a well-known liquid metal among the scientific community from thousands years before. But, due to its highly toxic nature, it is not recommended in electronic circuits. Therefore, other alternatives such as NaK alloy, ${ }^{85}$ galinstan $^{85}$ and stretchable silver conducting paste ${ }^{94}$ were proposed in literature. Galinstan is a liquid metal alloy of gallium, indium, tin and trace amounts of other elements. ${ }^{85}$ Among these, galinstan possesses significantly high electrical conductivity $\left(3.46 \times 10^{6} \mathrm{~S} / \mathrm{m}\right)$ and thermal stability $\left(-19^{\circ} \mathrm{C} \text { to } 1300^{\circ} \mathrm{C}\right)^{95}$ and is highly recommended for stretchable RF systems. A graphenebased flexible FET designed on PEN substrate has been reported in Ref. 90. The GFET shown in Fig. 4c can tolerate higher strain levels in comparison to a conventional FET fabricated on silicon thin films.

Elastomers have good mechanical flexibility, low thermal conductivity and a high CTE. Ceramics exhibit high permittivity and thermal conductivity. High processing temperature and brittleness restricts their application in flexible microelectronic industry. Butyl rubber and silicone rubber have excellent mechanical flexibility and stretchability. Elastomer ceramic composites integrate the high permittivity and high thermal conductivity of ceramic materials and low processing temperature, flexibility and stretchability of elastomers. Highpermittivity substrates are preferred for miniaturization of devices, whereas low-permittivity materials are used in high-speed devices. They are generally characterized by a low dielectric constant, low CTE and mechanical flexibility and stretchability. They don't undergo permanent deformation on bending. Hence, they are greatly recommended for stretchable electronics applications. Sebastian et al. investigated the microwave dielectric and mechanical and thermal properties of butyl and silicone rubber-based composites using several ceramic dielectrics of varying dielectric properties, and demonstrated fabrication of an antenna. ${ }^{96}$ Chen et al. investigated microwave absorption properties of graphene/silicon rubber composites. ${ }^{97} 1 \mathrm{wt} . \%$ holey graphene nanosheets are loaded in silicone rubber matrix and the resultant composite of $2-\mathrm{mm}$ thickness exhibited nearly $5 \mathrm{GHz}$ of absorption bandwidth.

A detailed investigation on microfluidic technology for stretchable electronics was done by Wu et al. in Ref. 85. Jung et al. reviewed various active and passive flexible and stretchable electronic components operating at high frequency. ${ }^{11}$ It was determined that an ideal substrate should exhibit high thermal stability, high dielectric constant and low loss tangent. But the flexible substrates used in the current technologies possess low dielectric constant, high loss tangent and comparatively low thermal conductivity. All these factors degrade RF performance due to dielectric loss and overheating of circuit components. Hence, more research activities should be encouraged in order to improve thermal stability and achieve lesser dielectric losses.

\section{Applications}

\section{Microwave Absorbers}

MWCNTs filled with Ni nanoparticles were incorporated into a polymer matrix of polystyrene. ${ }^{98}$ The resultant flexible composites are reported to have efficient microwave absorption properties. Polystyrene has been opted for as polymer matrix due to its low cost, light weight, high optical transparency and comparatively better thermal insulation. Lee et al. investigated the use of metamaterials in designing broadband microwave absorbers with a low radar cross section. ${ }^{99}$ The proposed microwave absorber shown in Fig. 4d consists of periodic metamaterial unit cells etched over polyamide substrate supported by a silicone rubber layer. It is found to be promising in military applications. The multiple layers of PET and PDMS were also used in designing metamaterial-based flexible microwave absorbers. ${ }^{100}$ Split ring resonators (SRRs) designed on conductive rubber are reported to function as mechanically tunable microwave absorbers. ${ }^{101}$ Conductive rubber is obtained by doping silicone rubber with silver nanoparticles. On bending along the symmetrical axis, the gap in SRRs increases; as a result, the effective capacitance decreases. Thus, the resonance frequency shifts to higher frequency on bending the structure along its symmetric axis. Zhang et al. designed a mechanically stretchable microwave absorber by adhering dielectric resonators of barium strontium titanate (BST) over a thin layer of conductive rubber as shown in Fig. 4e. On applying stress along the $\mathrm{H}$ field of the structure, coupling between adjacent unit cells varies, which in turn shifts the absorption peak. ${ }^{102}$

\section{Antenna Applications}

LCP has reported to find application in conformal antennas which can be conformed over surfaces of automobile or aircraft parts. ${ }^{103}$ It showed minimum distortion in RF performance on bending. Locher et al. proposed flexible textile microstrip patch antennas for Bluetooth applications. ${ }^{104}$ The radiating patch and ground were fabricated using silver and copper-coated fabric, whereas woolen felt or polyamide spacer fabric was used as substrate material. The proposed textile antenna maintained good radiation characteristics on bending. A stretchable UWB antenna designed using liquid metal alloy as the conducting material and PDMS as substrate material has been discussed in Ref. 95 . The liquid metal alloy is filled in designed channels in the flexible substrate. The proposed foldable and 
twistable antenna showed more than $70 \%$ radiation efficiency on stretching. It maintained good electrical performance up to $40 \%$ of stretching. Liyakath et al. proposed a stretchable multilayer conductor which consisted of a Zoflex layer sandwiched between two copper layers. ${ }^{105}$ It has been used in designing a frequency-reconfigurable antenna whose operating frequency is tuned by stretching along its length. Rizwan et al. fabricated a stretchable UHF RFID tag using the 3D printing technique. ${ }^{94}$ Stretchable silver paste and Ninja Flex were used as the conducting material and substrate, respectively. Liquid crystals are used to control the phase shift of elements in phased array by varying the DC voltage across the liquid crystal. ${ }^{106}$

\section{Electromagnetic Shielding}

A transparent and flexible thin film of PET coated with SWCNTs can be used as EMI shielding material at higher frequencies. ${ }^{107}$ PVDF is a flexible lightweight polymer suitable for electromagnetic shielding applications. A flexible polymer composite has been prepared by the addition of graphite and silver nanoparticles into PVDF matrix. ${ }^{108}$ Addition of conductive fillers like graphite and silver nanoparticles facilitates reflection of incident electromagnetic waves which in turn enhances shielding effectiveness.

\section{Biomedical Applications}

Flexible artificial tissue-mimicking materials are developed by mixing carbon black and graphite with urethane or silicone rubber. ${ }^{60}$ The polymer matrix provides flexibility, and the concentration of carbon powders controls permittivity and conductivity of the phantom material. Implantable antennas for neural recording systems are reported to be realized using Kapton polyimide as substrate material. ${ }^{109}$ The proposed antennas can be implanted efficiently in the brain as they are flexible and biocompatible.

\section{RF Integrating Devices}

High-speed thin-film transistors were realized using GaAs wires on PET and polyurethane substrate. ${ }^{110}$ It was found that the saturation current and on-to-off current ratio has minor variation with the increase in surface strain. Sun et al. proposed spiral inductors and capacitors designed over PET substrate for flexible microwave integrated circuit applications. ${ }^{111}$ On bending, the variation in inductance and capacitance was found to be within acceptable limits. LCP was identified as potential candidate for biocompatible devices due to its stability over long time and low moisture absorption property. Hwang et al. designed LCP-based flexible $\mathrm{RF}$ integrated circuits (RFICs) for implantable biomedical devices. ${ }^{112}$ Different techniques for the fabrication of flexible graphene and CNT-based thin-film transistors have been discussed in Ref. 113. The proposed methods are economical and employ fast fabrication techniques compared to other conventional techniques. Ma et al. have done an overall discussion on various flexible or stretchable microwave devices in Ref. 114; stretchable high-electron mobility transistors (HEMTs), flexible capacitors, flexible inductors, flexible filters and flexible transmission lines were realized on plastic or rubber material. The use of cellulose nanofibril paper as substrate for GaAs-based microwave devices has been explored in Ref. 115. Since cellulose nanofibril papers are biodegradable, it has been projected as a solution for toxic and nonbiodegradable electronic wastes. Gao et al. designed a flexible and miniaturized metamaterial by using ferrite thin film backed by polyimide material as substrate. ${ }^{116}$ The introduction of ferrite particles in the substrate increases capacitance and inductance which in turn reduces resonance frequency.

\section{Polarization Control Devices}

A metasurface designed over a flexible polyamide material simultaneously operates as a quarter-wave plate and beam splitter. ${ }^{117}$ A linearly polarized electromagnetic wave incident on the metasurface is transmitted and reflected as a circularly polarized wave in equal half-power and vice versa.

\section{Sensors}

Grenier et al. developed a flexible biosensor using PDMS, and the proposed sensor was able to discriminate between alive and dead biological cells. ${ }^{118}$ The designed RF sensor facilitated the interaction between electromagnetic waves and biological fluids. It works on the basis of shift in resonance frequency of the $R F$ resonator structure in the presence of fluid. Korostynska et al. developed a flexible microwave sensor to monitor water contaminants. ${ }^{119}$ The proposed real-time sensor was designed on polyamide laminate as shown in Fig. 4f. A flexible and stretchable transmission line-based pressure sensor has been proposed in Ref. 120. The transmission line deforms the substrate with variation in the applied pressure, thereby creating an impedance discontinuity which in turn causes reflection of the microwave input signal. Thus, this transmission line section functions as a pressure sensor.

\section{Waveguide Devices}

Bruno et al. designed flexible waveguides for millimeter-wave applications. ${ }^{121} \mathrm{~A}$ flexible waveguide fabricated using polytetrafluoroethylene (PTFE) of low dielectric constant has been filled with low-loss, high dielectric powders. The proposed waveguide was found to be suitable for shortdistance communication with comparatively low signal distortion. Kim et al. investigated the materials used for flexible cylindrical waveguides and 
the different losses associated with dielectric waveguides at millimeter-wave frequencies. ${ }^{122}$ Generally, dielectric materials having lower permittivity and dielectric losses such as PTFE, polypropylene, polyethylene and polystyrene are preferred for flexible waveguide applications. Yousef et al. fabricated a substrate-integrated waveguide (SIW) in flexible polyamide material for millimeter-wave applications. ${ }^{123}$ The estimated attenuation per unit length of SIW was comparable to that of microstrip lines at $79 \mathrm{GHz}$. The SIW-based slot showed a gain of $2.8 \mathrm{dBi}$ and front-to-back ratio of more than $15 \mathrm{~dB}$ at $78 \mathrm{GHz}$.

\section{Spintronic Devices}

Pinto et al. investigated the development of spintronic devices on flexible substrates. ${ }^{124}$ Magnetic tunnel junctions and a magnetic nanostructured pattern were successfully grown on flexible polyimide Kapton. It showed a high degree of flexibility without any performance degradation. Hence, Kapton can be used as substrate for flexible spintronic devices.

\section{BIOMATERIALS}

Nonbiodegradable materials are a great threat to the environment. The use of nonbiodegradable materials in $\mathrm{RF}$ devices and circuit components creates serious contamination in nature after their life span. The efficient disposal and recycling of electronic wastes have certain limitations. Recently, researchers have mainly focused on the development of ecofriendly material-based electronic devices using the available biomaterials in nature. Biocompatible and organic materials are recommended for implantable microwave devices in the biomedical field. Biomaterials were reported to find application in the development of biopolymers and natural fiberreinforced composites. Agricultural waste materials such as banana leaves $\left(\varepsilon_{r}=3.218, \tan \delta=1.014\right)$, rice husks $\left(\varepsilon_{r}=3.1, \tan \delta=0.827\right)$ and rice straw $\left(\varepsilon_{r}=2.492, \tan \delta=0.956\right)$ were reported to be used in designing microwave absorbers. ${ }^{125}$ Among the hexagonally shaped pyramidal absorbers designed using the above materials, banana leaf-based microwave absorbers showed better absorption over a wide bandwidth (1-20 GHz). Liyana et al. proposed sugarcane bagasse $\left(\varepsilon_{r}=1.44, \tan \delta=0.161\right)$ as an alternative material for designing pyramidal microwave absorbers for anechoic chamber applications. ${ }^{126}$ The pyramidal microwave absorber fabricated using sugarcane bagasse, polyester and methyl ethyl ketone peroxide (MEKP) contains only $9.3 \%$ chemical base material compared to other commercial microwave absorbers. It showed better absorption characteristics in the frequency range $0.1-20 \mathrm{GHz}$ compared to rice husk-based pyramidal absorbers. Seng et al. reported that the addition of CNTs into rice husk ash increases the dielectric constant and the loss tangent of the material. ${ }^{127}$ The CNT/rice husk composites are potential materials for designing of thin- and wideband microwave absorbers.

Bamboo charcoal has gained significant attention in the field of microwave absorbers due to its high electrical conductivity and self-lubricity. Wu et al. investigated the absorption characteristics of core shell magneto-electric composite containing bamboo charcoal. ${ }^{128} \mathrm{CoFe}_{2} \mathrm{O}_{4} /$ bamboo charcoal/titania/LDPE magneto-dielectric polymer composites are also reported in literature. ${ }^{129}$ Bamboo charcoal composite showed a permittivity of 2.272 at $9.94 \mathrm{GHz}$. The inclusion of bamboo charcoal in the polymer matrix causes multiple scattering of electromagnetic waves, thereby increasing its absorption characteristics. Another bamboo-based magneto-dielectric composite was synthesized by mixing NiZn ferrite, bamboo charcoal and titania particles in low-density polyethylene. ${ }^{130}$ The proposed composite showed wide absorption over the X-band. Guna et al. designed and successfully fabricated a completely biodegradable printed circuit board using bio-composites. ${ }^{12}$ The bio-composites were prepared using wheat gluten and natural fibers extracted from banana stem. Even though it has comparatively low flexural and tensile strength (27 MPa and $45 \mathrm{MPa}$, respectively), its density is low $\left(1.03 \mathrm{~g} / \mathrm{cm}^{3}\right)$ compared with $1.85 \mathrm{~g} / \mathrm{cm}^{3}$ that of FR4.

Carbon-based materials such as carbon black, CNTs, graphite and activated carbon are characterized by high electrical conductivity and low density. They are promising materials for the fabrication of lightweight microwave absorbers at higher frequencies. Rice husks, coconut shell, sugarcane bagasse, dried banana leaves, oil palm leaves and bamboo are natural carbonaceous materials. They are used to produce activated carbon which finds wide application in cost-effective microwave absorbers. Salleh et al. developed a lightweight and cost-effective radar-absorbing material using coconut shell-based activated carbon mixed with flaxane- 80 binder. ${ }^{131}$ The developed absorber contains a single layer of activated carbon backed by a metallic sheet as shown in Fig. 5a. The prototype of 7.5-mm thickness containing $25 \%$ of carbon exhibits more than $1 \mathrm{GHz}$ of absorption bandwidth. Rubber wood saw dust has been chemically treated with $\mathrm{ZnCl}_{2}$ followed by heating to obtain activated carbon. The activated carbon had a high surface area of $1301 \mathrm{~m}^{2} / \mathrm{g}$ and it is dispersed in polyurethane rubber. ${ }^{132}$

\section{Applications}

\section{Microwave Absorbers}

Rice husks have been used as base material for designing wedge-shaped absorbers, ${ }^{133}$ pyramidal absorbers $^{134}$ and metamaterial-based pyramidal absorbers. ${ }^{135}$ Agro-waste materials such as dried banana leaves mixed with polyester and MEKP ${ }^{136}$ were also used for designing pyramidal microwave 


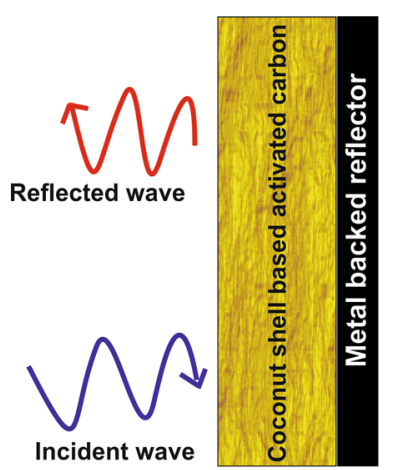

(a)

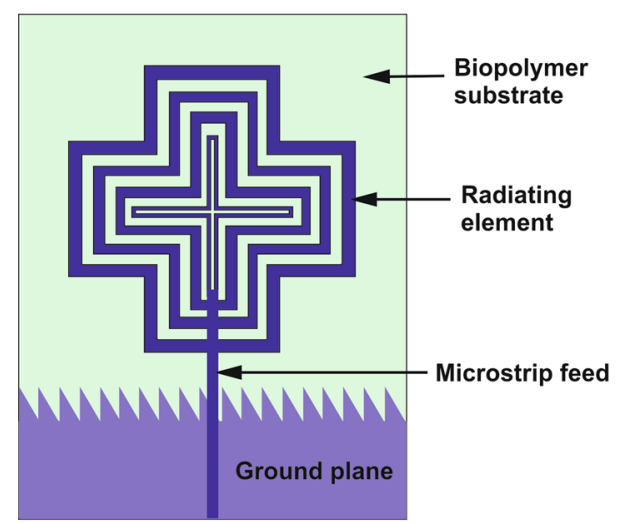

(c)

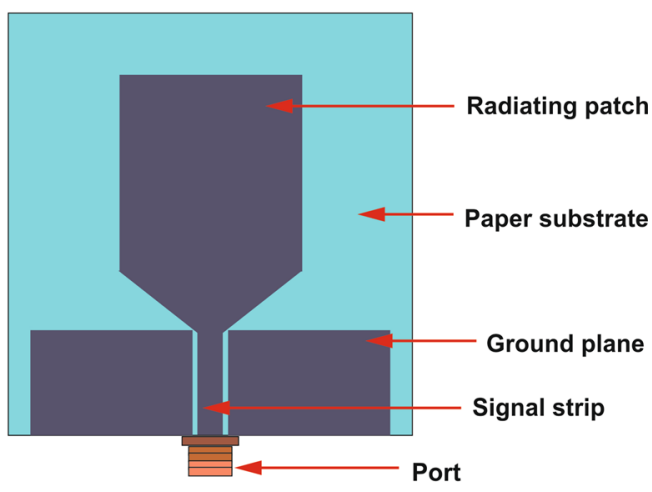

(b)

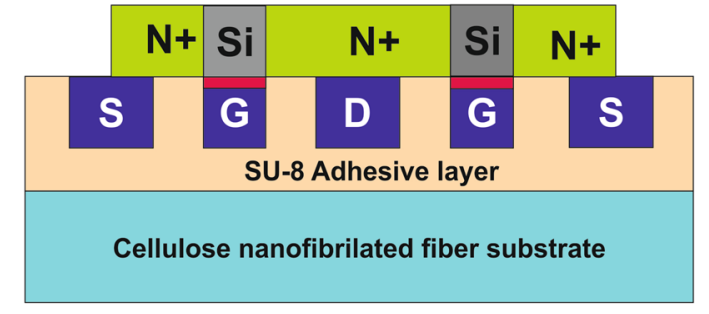

(d)

Fig. 5. Microwave devices using biomaterials: (a) coconut shell-based microwave absorber (modified and redrawn using the data from Ref. 131), (b) paper-based UWB antenna (modified and redrawn using the data from Ref. 140), (c) antenna on rice husk-based biopolymer substrate (modified and redrawn using the data from Ref. 141) and (d) flexible transistor on cellulose nanofibrilated fiber substrate adapted with permission from Ref. 143, copyrighted by AIP Publishing.

absorbers. Fine particles of agricultural wastes such as oil palm leaves, rice husks and empty fruit bunches have shown significant absorption of incident electromagnetic waves. ${ }^{137}$ In 2015 , Jin et al. demonstrated microwave absorption properties of bamboo-based magnetic composites. ${ }^{138}$ The proposed composite was developed by depositing $\gamma-\mathrm{Fe}_{2} \mathrm{O}_{3}$ nanoparticles on bamboo material. It showed nearly $70 \%$ absorption over the X-band and an excellent water-resistant property.

\section{Electromagnetic Compatibility}

Iqbal et al. reported the use of a mixture of powdered rice husk and a nontoxic resin as a microwave absorber to suppress electromagnetic interference in electronic systems. ${ }^{139}$ They studied the dielectric properties of the material in the frequency range $4-8 \mathrm{GHz}$ with different composition of rice husk powder.

\section{Antenna Applications}

Paper is a cost-effective and eco-friendly substrate for many printable microwave devices. An
UWB antenna printed on commercially available paper has been discussed in Ref. 140. The geometry of the proposed antenna is illustrated in Fig. 5b. The monopole antenna realized on paper substrate shows more than $80 \%$ efficiency and less than 0.6-nS variation in group delay, which ensures minimum distortion of transmitted or received signals in the operational band. Ullah et al. demonstrated the design and development of an UWB microstrip patch antenna on biomaterial-based substrate. ${ }^{141}$ The geometry of the aforementioned antenna is shown in Fig. 5c. The proposed organic substrate was prepared from aerogel nanoparticles which were extracted from rice husks and organic polybutylene succinate (PBS). The designed biomaterial substratebased antenna shows good radiation performance over the UWB spectrum. Jute has been reported as substrate material for textile antennas. A dual-band antenna with an omnidirectional pattern and sufficient bandwidth is designed on jute for wireless local area network (WLAN) applications. ${ }^{142}$ 


\section{Microwave Devices}

Cellulose nanofibril extracted from wood was proposed as a potential microwave substrate for different $\mathrm{RF}$ and digital electronic devices by Jung et al. ${ }^{115}$ It shows high optical transparency, mechanical flexibility and desired electrical properties. Cellulose nanofibers are biodegradable, flexible and have low thermal expansion. A thin-film transistor realized on cellulose substrate is depicted in Fig. 5d and it shows a higher cut-off frequency of $10.6 \mathrm{GHz} .{ }^{143}$ Zeouga et al. investigated the use of jute as microwave substrate for designing the rectifier part of a rectanna system. ${ }^{144}$ Better efficiency and the rejection of higher-order harmonics have been achieved while using multilayered jute material.

\section{Biomedical Applications}

Bindu et al. investigated the dielectric properties of different concentrations of corn syrup and found that it can be used as promising material to mimic the dielectric properties of breast tissues. ${ }^{145}$ They proposed that corn syrup can be used as an effective coupling medium in microwave imaging to reduce scattering which in turn enhances resolution.

\section{Sensors}

Bahoumina et al. designed a chemical sensor to monitor environmental pollution. ${ }^{80}$ The proposed ink jet printable sensor designed on paper consisted of a sensitive carbon composite layer. It works on the principle of change in capacitive resonator frequency on the absorption of chemical vapors.

\section{ELECTROMAGNETIC PHANTOM MATERIALS}

The rapid increase in wireless devices causes interaction between an electromagnetic field and the human body, especially at microwave frequencies. The interaction can be of two different types; biological effect of radiations on human body and the influence of human body in the performance of wireless devices. ${ }^{146}$ But testing the electromagnetic effects directly on living tissues is not allowed due to ethical concerns; as such, simulated biological models termed as phantoms are usually used for such electromagnetic studies. Phantoms may be defined as a body-equivalent tissue mimicking the electrical properties of a biological tissue. Biological tissues are characterized by their dielectric properties and conductivity at a specific range of temperature and frequency. Gabriel et al. calculated the electrical properties of body tissues in the frequency range of $10 \mathrm{~Hz}-100 \mathrm{GHz}$ and the data is available in the Italian National Research Council website ${ }^{147}$ which is used as a standard reference for the fabrication of phantoms. The major requirements of phantom materials are; it should be nonhazardous to humans or other materials under test, homogenous, of low cost and easier fabrication, flexible to fit various setups and should maintain stable electrical and thermal characteristics over a wide band of frequency to ensure repeatable measurements. ${ }^{146,148}$

\section{Classifications}

The composition and design of a particular phantom depends on its application. Therefore, phantom materials can be classified as solid (dry), semisolid (gel) and liquid phantoms based on their physical nature. Based on the type of tissue under investigation, there are high-water-content tissues, with high permittivity and loss tangent and low-watercontent tissues with low permittivity and low loss tangent. Muscles, brain and skin are high-watercontent tissues, whereas bones and fats come under the category of low-water-content tissue. ${ }^{149}$ A photograph of a multilayered skin phantom is shown in Fig. 6.

Liquid phantoms have certain advantages, easier preparation by mixing different components based on dielectric properties of emulating tissue and they

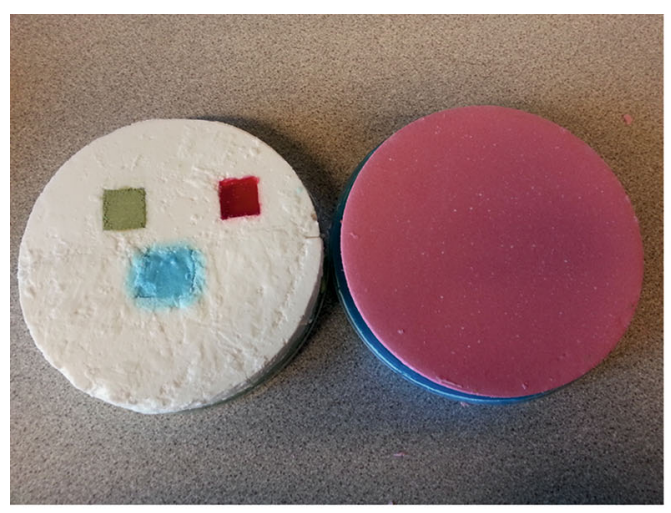

(a)

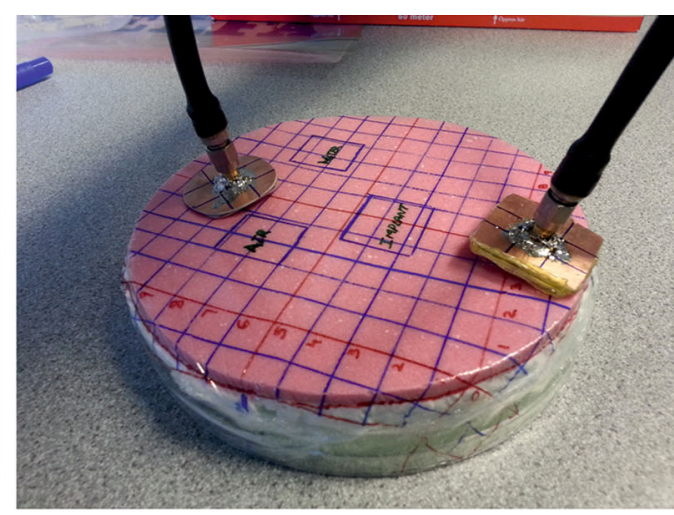

(b)

Fig. 6. Photographs of phantom materials: (a) multilayer skin phantom for monitoring surgical healing and (b) skin phantom under dielectric measurement. 
facilitate direct measurement of electric field inside the phantom using electric field probes. Usually, different proportions of sugar and diacetin or diethylene glycol butyl ether (DGBE) are used to control dielectric properties at lower and higher frequencies, respectively. ${ }^{149}$ Sodium chloride $(\mathrm{NaCl})$ is used to increase ionic conductivity, but it has no influence above $25 \mathrm{GHz} .{ }^{150}$ Addition of sucrose to the solution reduces relative permittivity, but slightly increases conductivity. ${ }^{150}$ But the dielectric property of the liquid container affects the accuracy of permittivity measurement. Oil-in-water emulsions are generally used to mimic low-water-content tissues in which polysorbate or TX-100 are used as emulsifying agents. ${ }^{150}$

Agar, gelatine and TritonTX-151 are generally used as gelling agents to obtain a desired shape in semisolid phantoms. ${ }^{150}$ Even though conventional gel phantoms are flexible, they possess certain drawbacks such as dehydration and deterioration due to bacterial or fungal growth which puts a limit on maintaining the material properties for a long time. A phantom shell helps in retaining a shape and limits dehydration to a certain extent. But, the necessity of a phantom shell affects the accurate estimation of surface electric field distribution inside the biological organs. Distilled water is the fundamental solvent in most of the phantoms. Recently, it has been reported that vinegar can be used as a preservative for a gelatine-based phantom since it doesn't change electrical properties significantly. ${ }^{151}$ The sample with $50 \%$ of vinegar has a shelf life of 6 weeks, but it becomes unstable as the solidity decreases with the increase of vinegar content. Preservatives such as sodium azide ${ }^{150}$ and formaldehyde ${ }^{151}$ can be used to enhance the shelf lifetime of liquid and gel phantoms. But, the toxic nature of formaldehyde limits its use as bactericide.

Solid phantoms overcome some issues associated with liquid and gel phantoms, since they are capable of retaining shape and material properties over a long period of time. Hence, it is possible to perform precise and reliable estimation of surface field distribution in biological systems. Kobayashi et al. proposed a dry phantom containing ceramic powder, graphite powder and resin. ${ }^{152}$ They observed that the proposed dry phantom maintains the same dielectric properties over UHF band. But the use of adhesive materials to avoid air gaps in traditional ceramic phantoms makes them heavy and difficult to be reshaped. Graphite powder coated with bonding resin such as polyvinylidene fluoride is used to increase conductivity in solid phantoms, whereas polyvinyl chloride (PVC) powder is used to decrease conductivity. ${ }^{150}$ Silicone rubber is used as base material to fabricate soft solid phantoms. A soft and dry phantom composed of carbon fibers and silicone rubber has been proposed in Ref. 153. Highwater-content and low-water-content tissues can be emulated by controlling the weight ratio of carbon fibers in the sample. Hikage et al. fabricated a lightweight solid phantom by mixing carbon black and CNTs with silicone rubber and, finally, a realistic human phantom model has been constructed using the proposed material. ${ }^{154}$ The composition ratio of carbon black and CNTs in silicon rubber is controlled to mimic human muscle tissues. They observed that the amount of CNTs and carbon black mainly controls the relative permittivity and loss tangent of the phantom, respectively.

A heterogeneous breast phantom consisting of skin, fat, gland and tumor tissues has been realized using water and oil as main ingredients. The detailed recipe has been explained in Ref. 155. Oil in gelatine-based mixtures and a mixture of distilled water, Triton X-100 (TX-100) and salt are used to fabricate low-permittivity artificial tissues like breast phantom for experimental validation of microwave imaging systems. ${ }^{156}$ The TX-100-based phantoms are easily reproducible and have stable electrical properties over temperature and time period compared to oil in gelatine-based phantoms.

Today, many commercial electromagnetic simulation software packages are available with numerical phantom models, and they can be used to measure biological effects on living tissues caused by mobile antenna terminals or other wireless devices. But, the software has some limitations in trouble-shooting hardware-level implementations. A detailed discussion on different artificial phantoms and a list of reported phantoms emulating different body parts such as head, limbs, breast and torso phantoms have been given in Ref. 150 .

\section{Applications}

Phantoms have significant applications in wireless communication as well as microwave biomedical systems such as performance evaluation of wireless devices, microwave imaging techniques and therapeutic devices, thus ensuring quality of services. They also have some military and space applications, including performance evaluation of battle field personal care and smart suits for astronaut monitoring. ${ }^{149}$

\section{Biomedical Applications}

Microwaves, being nonionizing radiations, can be used in medical imaging and different therapeutic techniques to reduce radiation hazards. Augustine et al. fabricated a craniotomy phantom to analyze healing of bone implants in post-craniosynostosis surgery. ${ }^{157}$ The developed body-equivalent tissue which consists of three layers of skin, bone and brain phantoms helps in the design of antennas for monitoring surgical healing. A realistic head phantom consisting of the main tissues of brain (hair, scalp, skull, cerebrospinal fluid, and grey and white matter) has been reported in literature to test brain imaging systems. ${ }^{158} \mathrm{~A}$ compact antenna has been designed for capsular endoscopes with a wireless 
power transmission system. ${ }^{159}$ It can diagnose tumor growth in digestive organs including the small intestine. The antenna performance evaluation has been done using an abdomen phantom consisting of muscles and other internal organs. Wearable antennas are essential for constant monitoring of patients under emergency conditions. A dual-mode antenna capable of efficiently transmitting biological information such as heart beat has been developed by Ito et al.; the experimental evaluation can be done by using torso phantoms, and they have developed a biocompatible RFID tag antenna for in-body wireless communication which can be implanted permanently in the human body. ${ }^{159}$ It reduces chances of medical errors through constant monitoring of patients. A multilayer phantom simulating a real human hand was used to measure the performance of the designed system. The design and development of walled carotid bifurcation phantoms has been discussed in Ref. 160. The walled structure with high elasticity and acoustic capability provides means of imaging wall motion and blood flow dynamics simultaneously, which is useful in vascular disease diagnosis. Hattenhorst et al. developed a flexible phantom using barium titanate and potting compound for testing three microwave techniques; microwave tomography, radar ellipsometry and coaxial probing. ${ }^{148}$ Joachimowicz et al. reported the development of different anthropomorphic phantoms using 3D printing, and the fabricated structures were filled with liquids. ${ }^{161}$ Performance of microwave imaging systems can be estimated using these phantoms. Carbon-based rubber phantoms which emulate dielectric properties of different tissues like skin, fat, glands and tumors have been developed by Moll et al. ${ }^{162}$ They are easy to fabricate and have stable electrical properties which are suitable for microwave biomedical applications.

\section{Wireless Communications}

Phantoms play a significant role in the design and testing of implantable devices in order to assess the electromagnetic interaction with human bodies. A semisolid phantom has been fabricated for the visualization and estimation of $3 \mathrm{D}$ distribution of local SAR. ${ }^{163}$ The proposed phantom containing gellan gum or polyacrylamide, non-ionic surface active agent and salt has good optical transparency at room temperature and becomes locally white and opaque on absorbing electromagnetic energy. ${ }^{163}$ Further modification has been done in the same phantom model by avoiding the necessity of a container. ${ }^{164}$ Radiation risk of mobile phones is assessed by measuring SAR value. The recipes of different liquid phantoms which can be employed for the safety evaluation test of mobile phones have been discussed in Ref. 165. Okano et al. developed a brain phantom and skull phantom for the measurement SAR. ${ }^{166}$ A brain-equivalent phantom has been synthesized using deionized water, agar, $\mathrm{NaCl}$, sodium azide, polyethylene powder and TX-151. $\mathrm{NaCl}$ contributes to the loss tangent, and polyethylene is used to control permittivity of the tissue. Sodium azide is used as preservative. The recipe for a skull phantom mainly consists of glycerol and silicone emulsion. Since skull tissues are characterized by a low loss tangent, glycerol is the best solvent due to its low permittivity. Felicio et al. used a multilayer phantom and homogeneous liquid phantom to compare the performance of an UWB antenna. The measurements showed that a simple homogeneous phantom can replace the use of multilayer phantom without affecting performance seriously. ${ }^{167}$

\section{TUNABLE MATERIALS}

Tunable microwave materials are widely used for various microwave applications such as phase shifters, tunable capacitors, delay lines and filters. They have great demand in the field of wireless communication as they provide compact and lightweight integration devices with high tunability and low insertion loss. Tunability is a measure of change in dielectric or magnetic properties of a material in the presence of an applied electric or magnetic field. The propagation of electromagnetic waves in a material is generally characterized by their complex permittivity and permeability. If the permittivity and permeability of a material can be controlled by an external electric field or magnetic field, or both, or mechanical force, such materials are termed as tunable materials. The molecules inside the material align according to the applied electric or magnetic field direction. It can be utilized for numerous microwave applications such as controlling the phase shift in antenna arrays and phase shifters, capacitance in varactors, resonance frequency of antennas and filters, etc. External magnetic field tuning systems are generally bulky and incompatible for integrated circuits with low tuning speed and high power consumption. Therefore, electrically tunable materials are preferred as they have high tuning speed, comparatively low power consumption and can be easily integrated with other RF components.

Ferroelectric materials are the most investigated electrically tunable materials for microwave applications due to higher tunability and lower power consumption. Ahamed et al. have done an excellent review on electrically tunable materials for microwave application. ${ }^{13}$ Bulk ferroelectric materials have more losses and large value of permittivity which strongly depend on temperature. It is desirable for ferroelectric materials to operate just above Curie temperature in order to achieve great tunability and less loss. Ferroelectric thin films need lesser voltage for tunability compared to bulk material. ${ }^{13}$ Barium titanate is mixed with strontium titanate in order to reduce its Curie 


\section{Table I. Materials for tunable applications}

\begin{tabular}{|c|c|}
\hline Applications & Material \\
\hline $\begin{array}{l}\text { Tunable microwave oscilla- } \\
\text { tors }\end{array}$ & Yttrium iron garnets $(\mathrm{YIGs})^{18}$ \\
\hline Tunable phase shifters & YIG ${ }^{19}$ multilayered NiFe thin films and YIG ferrite ${ }^{191}$ \\
\hline Tunable filters & $\begin{array}{l}\text { Yttrium iron garnets and Al-doped YIG ferrite, }{ }^{37} \text { liquid crystal, }{ }^{183} \mathrm{BST} \text { thin films doped } \\
\text { with Mn, }{ }^{184} \text { soft ferromagnetic nanoparticles } \mathrm{Fe}_{60} \mathrm{Co}_{40},{ }^{184} \mathrm{YIG}^{, 37} \text { Al-doped } \mathrm{YIG}^{37}\end{array}$ \\
\hline Tunable capacitors & BST thin films ${ }^{169,178}$ ATN thin films ${ }^{180}$ BZN thin films,${ }_{181}^{181}$ PST thin films ${ }^{182}$ \\
\hline Tunable inductors & Permalloy thin films ${ }^{187}$ \\
\hline $\begin{array}{l}\text { Tunable microwave absor- } \\
\text { bers }\end{array}$ & Graphene, ${ }^{68}$ dielectric resonators of BST over a thin layer of conductive rubber ${ }^{102}$ \\
\hline $\begin{array}{l}\text { Frequency reconfigurable } \\
\text { antennas }\end{array}$ & $\mathrm{Co}_{2} \mathrm{Z}$ hexa ferrite, ${ }^{31} \mathrm{FeCo}$ nanoparticles or thin films, ${ }^{35} \mathrm{Y}_{3} \mathrm{Fe}_{5} \mathrm{O}_{15}{ }^{192}$ \\
\hline $\begin{array}{l}\text { Pattern reconfigurable } \\
\text { antennas }\end{array}$ & Multilayered lead zirconate, YIG and gadolinium gallium garnet, ${ }^{36}$ liquid crystal ${ }^{189}$ \\
\hline $\begin{array}{l}\text { Polarization reconfigurable } \\
\text { antennas }\end{array}$ & Liquid crystals, ${ }^{106} \mathrm{YIG}^{192}$ \\
\hline
\end{tabular}

temperature. The resulting barium strontium titanate (BST) is one among the well-known tunable microwave materials at room temperature. BST compounds exhibit certain advantages like lower power consumption, high power handling, high tuning speed and continuous tuning. ${ }^{168}$ BST ceramics and thin films with higher dielectric susceptibility have found applications in phase shifters, dielectric resonators and capacitors. A moderate dielectric constant and the temperature stability of permittivity and high tunability are essential for these applications. The dielectric losses of BST ceramics can be reduced by the addition of dopants such as $\mathrm{TiO}_{x},{ }^{169} \mathrm{Bi}^{170} \mathrm{Fe},{ }^{170} \mathrm{Mg}^{170}$ and $\mathrm{Mn} .{ }^{171} \mathrm{~A}$ method for developing barium-strontium-calciumtitanate ceramics with dielectric temperature stability and tunability has been proposed in Ref. 172 . Addition of $\mathrm{Ca}$ to BST ceramics reduces the dielectric constant and enhances temperature stability by maintaining high tunability.

Another perovskite used for microwave tunable application is lead strontium titanate (PST). It is obtained by the addition of lead titanate into strontium titanate, and its Curie temperature can be controlled by concentration of lead and strontium. ${ }^{13}$ But due to the toxicity of lead, PST is not highly recommended for tunable applications in electronic circuits. Silver tantalite niobate (ATN) and barium stannate titanate (BTS) are the other commonly used tunable ferroelectric materials. ${ }^{13}$ ATN has lower tunability compared to other ferroelectric materials, but BTS has excellent tunability, low loss, wide operating temperature and frequency stability. ${ }^{13}$ Bismuth zinc titanate (BZN) is a nonferroelectric material having higher tunability and BZN thin films have very low dielectric losses. Organic polymers such as polyvinylidene fluoride (PVDF) and its co-polymers are also used for electrically tunable applications. ${ }^{13}$ Even though they are flexible and lightweight, they are expensive and more sensitive to temperature variations ${ }^{13}$ Liquid crystals are new emerging class of materials for tunable microwave applications. Since they are anisotropic in nature, external electric field can change the orientation of the molecules which in turn varies dielectric constant. ${ }^{13}$ It provides continuous tuning at higher frequencies. CNTs show semiconductor or metallic nature according to their electronic structures. They are also used in electrically tunable microwave devices. ${ }^{173}$ Zhao et al. investigated shielding effectiveness of PVDF/Ni chain composites. ${ }^{174}$ The shielding effectiveness can be tuned by changing the height of the composite. Ni nanoparticles are comparatively cost-effective compared to usual carbon fillers used in polymer composites.

It is easy to control the magnetic moment in ferromagnetic materials with an external magnetic field as compared with anti-ferromagnetic materials. $\mathrm{Fe}_{2} \mathrm{O}_{3}$ nanoparticles uniformly dispersed in a polymer matrix were used to realize a microwave substrate exhibiting super-paramagnetism or lack of hysteresis losses. ${ }^{175}$ The permittivity, permeability and loss tangents of the substrate can be changed by an external magnetic field. A bilayered composite material consisting of a ferromagnetic layer of $\mathrm{FeCoSiB}$ and piezoelectric substrate of lead zirconate titanate (PZT) can be used for tunable microwave applications as the permeability of this magnetostrictive/piezoelectric composite varies with different DC voltages. ${ }^{176}$ Ferromagnetic microwires exhibit giant magneto impedance, low coercivity and high saturation magnetization. Glass-coated ferromagnetic microwires are reported to be suitable for designing microwave energy sensors based on their microwave absorption properties. ${ }^{177}$ Some important materials used for various microwave applications are listed in Table I. It includes both magnetically and electrically tunable microwave materials and their applications extend from 


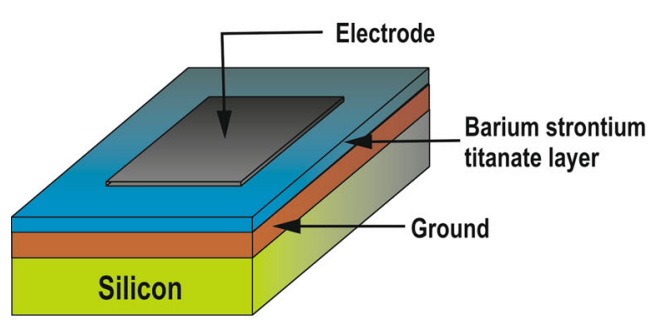

(a)

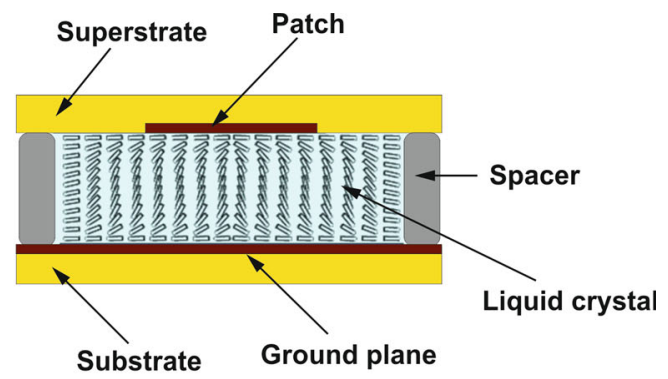

(b)

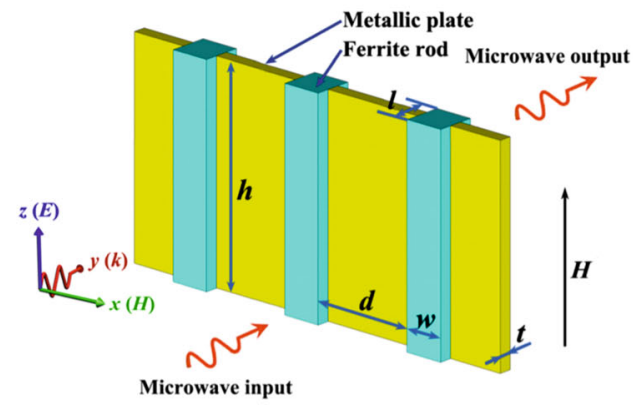

(c)

Fig. 7. Microwave devices using tunable materials. (a) BST-based tunable thin-film capacitor (modified and redrawn using the data from Ref. 178), (b) single element of tunable liquid crystal reflect array antenna (modified and redrawn using the data from Ref. 188) and (c) magnetically tunable band-pass filter using ferrite rods adapted with permission from Ref. 193, copyrighted by AIP Publishing.

tunable devices such as tunable capacitors and tunable inductors for microwave integrating circuits to microwave absorbers and antenna applications.

\section{Electrically Tunable Applications}

\section{Tunable Capacitors}

A planar BST thin-film capacitor shown in Fig. 7a is fabricated using the metal organic chemical vapour deposition method. ${ }^{178}$ The high dielectric constant of BST thin films can be tuned by applying low DC voltages. Hence, BST films with low loss tangents are suitable for the fabrication of capacitors with high capacitance density. The proposed capacitor shows greater tunability and Q factor compared to commercially available varactor diodes of the same capacitance. BST thin film-based capacitors can be used in the design of tunable LC ladder type phase shifters. ${ }^{179}$ Bonetti et al. designed two types of varactors using ATN thin films; buried capacitor structures and capacitors etched on top of the film. ${ }^{180}$ On comparing the performance of the two varactors, buried varactors showed a high dielectric constant and greater tunability than conventional topped varactors. Lee et al. designed inter-digital capacitors with higher tunability using BZN thin films. ${ }^{181}$ The electrodes of the capacitor are embedded into the BZN thin films fabricated on silicon substrate which reduces biasing voltages and increases tunability. PST thin-film-based varactors were designed on high-resistivity silicone substrate to reduce conductive losses in the substrate. ${ }^{182}$
Later, these varactors were used to design tunable band-stop filters and resonators. BST film with (111) orientation shows higher dielectric tunability as compared to (001)-orientated films. ${ }^{169}$ Addition of dopants in thin film introduces structural defects, so a buffer layer of $\mathrm{TiO}_{x}$ with optimized thickness has been used to obtain (111)-oriented BST films which were synthesized on $\mathrm{Al}_{2} \mathrm{O}_{3}$ substrate of low losses. ${ }^{169}$ They can be used to fabricate interdigitated capacitors.

\section{Tunable Filters}

Follmann et al. proposed liquid crystal-based tunable filters for space applications. ${ }^{183}$ Dielectric permittivity can be tuned by applying external magnetic or electric field. The resonance frequency of the designed filter was shifted in accordance with the change in permittivity. Ferroelectric thin films of BST doped with manganese can be used to design light and compact tunable filters due to an electrically tunable high dielectric constant and low loss of the material. ${ }^{184}$

\section{Tunable Varactors, Inductors and Phase Shifters}

A discrete phase shifter has been designed by employing BZN thin-film-based capacitors. The designed phase shifter shows low insertion loss and better tunability due to discrete capacitive loading. ${ }^{185}$ Ferroelectric BST ink is developed by mixing nano- or micro-sized BST particles with thermoplastic cyclic olefic copolymer. Fully printed 
electrically tunable varactors and phase shifters were realized using this dielectric ink. ${ }^{186}$ Permalloy (Py) is a ferromagnetic material with large permeability, low coercivity, small magnetostriction and stress anisotropy. A fully electrically tunable inductor with high inductance and quality factor can be fabricated using patterned Py thin films. ${ }^{187}$ A slow wave transmission line has also been developed by integrating both Py films and ferroelectric PZT which finds applications in designing phase shifters. ${ }^{187}$ By the application of DC voltage and current the capacitance and inductance can be tuned simultaneously, keeping the characteristic impedance as a constant.

\section{Antenna Applications}

Bildik et al. designed a tunable reflect array and the schematic of the single antenna element of the proposed array is shown in Fig. 7b. It consists of rectangular patches on the lower side of the superstrate and liquid crystal is placed between the ground plane and patch. ${ }^{188}$ On applying DC voltage, orientation of the molecules changes in the liquid crystal which in turn changes the permittivity. Thus, electrically tunable permittivity can be used to obtain different phases to tune the reflect array. A polarization reconfigurable microstrip dual-fed array has been designed and the desired phase shift is obtained using tunable liquid crystal material. ${ }^{106}$ Linear or circular polarization can be achieved by biasing liquid crystal material placed between upper and lower dielectric substrates. Roig et al. proposed a tunable liquid crystal-based leaky wave antenna, and continuous tuning of radiation angle is achieved by applying bias voltage across the liquid crystal. ${ }^{189}$ The leaky wave antenna operates at $27.6 \mathrm{GHz}$, and the main beam can be steered from $-5^{\circ}$ to $9^{\circ}$ by varying the applied voltage from 0 $\mathrm{V}$ to $60 \mathrm{~V}$.

\section{Tunable Microwave Absorbers}

Deng et al. designed a tunable metamaterial absorber on liquid crystal. ${ }^{190}$ It consists of a liquid crystal layer sandwiched between a metal pattern and ground plate. The whole structure is kept between quartz plates. Tunability is achieved by applying DC voltage across the metal pattern and the ground. It resonates at $110.85 \mathrm{GHz}$ under unbiased condition and at $9 \mathrm{~V}$ resonance shifts to $103.8 \mathrm{GHz}$. The absorption peak shifts towards lower frequency as the bias voltage increases.

\section{Magnetically Tunable Applications}

\section{Tunable Phase Shifters}

Two magnetically tunable phase shifters are fabricated using multilayered NiFe thin films and YIG ferrite as active media. ${ }^{191}$ The YIG ferritebased resonance phase shifter shows superior characteristics due to its low magnetic loss tangent. Ferromagnetic resonance can be controlled by a comparatively low magnetic field which enables the integration in RF circuits.

\section{Antenna Applications}

A ferroelectric component and a magnetic component co-exist in multiferroic materials, thus enabling the tuning of resonance frequency with an external electric or magnetic field, respectively. Ferrimagnetic compound $\mathrm{Y}_{3} \mathrm{Fe}_{5} \mathrm{O}_{12}$ having a Curie temperature of $550 \mathrm{~K}$ can be used as antenna substrate. ${ }^{192}$ The resonance frequency and polarization of the proposed antenna can be changed by an external magnetic field.

\section{Tunable Filters}

Soft ferromagnetic nanoparticles like $\mathrm{Fe}_{60} \mathrm{Co}_{40}$ find application in the design of tunable filters. ${ }^{184}$ Magnetically tunable band-pass filters have been realized using ferrite rods. ${ }^{193}$ The geometry of the prototype using ferrite rods arranged inside metallic slits is illustrated in Fig. 7c. Metallic walls inhibit the penetration of microwave signals and the ferrite rods filled inside the metallic slits show ferromagnetic resonance with the applied magnetic field. It results in enhanced electromagnetic energy transmission, and the transmission response can be tuned by an external magnetic field. Microwave band-pass filters operating in $\mathrm{C}$ band are fabricated using YIG and Al-doped YIG material. ${ }^{37}$ It shows lower insertion loss and better bandwidth. The tunability of filters depends on the magnetization of materials under an external magnetic field.

\section{METAMATERIALS}

Metamaterials are artificially engineered materials exhibiting exceptional properties that are not observed in natural material or in their constituent materials at the frequencies of interest. The International society on metamaterial existing worldwide, 'Virtual Institute of Artificial Electromagnetic Materials and Metamaterials,' defines it as an arrangement of artificial structural elements designed to achieve advantageous and unusual electromagnetic properties. ${ }^{194}$ A detailed discussion on the proper definition of metamaterials has been done by Ari Sihvola. ${ }^{195}$ A metamaterial can be tailored by a proper spatial arrangement of inclusions within a host material. Electromagnetic response of a metamaterial depends on the geometry and alignment of inclusions, composition as well as the lattice constants of its structural unit. ${ }^{196}$ Metamaterials are used for various applications such as electromagnetic cloaking, sub-wavelength imaging, metamaterial-based sensors, antenna downsizing, enhanced power transmission through sub-wavelength apertures and electromagnetic shielding. 
The concept of artificial permittivity has been explored from days of J.C. Bose, who used manmade twisted fibers (jute) to rotate the polarization of electromagnetic waves. ${ }^{197}$ Although some earlier works have suggested the existence of backward waves, it was Mandelstam who discussed negative group velocity and the negative index of refraction in $1945 .{ }^{198}$ But a systematic theoretical study on general properties of wave propagation in negative refractive index medium has been done by Russian physicist V.G. Vesalago in 1967 and he called such materials as left-handed media (LHM) ${ }^{199}$ Sir J. Pendry demonstrated that the arrays of metallic resonators of sub-wavelength dimensions exhibits negative permeability for a certain frequency band when excited with electromagnetic waves. ${ }^{200}$ In 2001, Shelby et al. gave the experimental verification of the negative index of refraction on a structured metamaterial at microwave frequencies. ${ }^{201}$ The possibility of developing electromagnetic materials with arbitrary values of $\varepsilon$ and $\mu$ gave a new dimension to material engineering, and the unique characteristics of these materials could be beneficial in wireless and optical communications.

Metamaterials can be used to guide or manipulate electromagnetic waves, which depend on the material parameters $\mu$ and $\varepsilon$. There are mainly five classes of materials when the sign of these quantities are taking into consideration. A detailed discussion on different categories of metamaterials has been given in Ref. 202. The materials having a positive real part of permittivity and permeability are called double-positive (DPS), as most materials found in nature. If both these quantities are negative, they are called double-negative (DNG) media. Materials of this category are of great research interest. DNG media can be realized artificially, as they are not available in nature. The first successful development of a metamaterial exhibiting a negative refractive index was reported in 2001. The material was composed of a periodical two-dimensional array of copper strips and SRRs on interlocking strips of a standard circuit board. ${ }^{201}$ Media having a negative real part of permittivity and those having a negative real part of permeability may be named as $\varepsilon$-negative (ENG) and $\mu$-negative (MNG) media, respectively. They may be collectively termed as single-negative media (SNG). MNG materials were realized using microstructures developed from non-magnetic conducting sheets. ${ }^{200}$ Plasma and plasmonic materials under their plasma frequencies show the characteristics of ENG media. In case of zero-index materials, both permittivity and permeability are zero. Materials with a real part of permittivity or permeability having low value (near to zero) are termed as $\varepsilon$-near-zero (ENZ) and $\mu$-nearzero (MNZ) materials, respectively. ${ }^{202}$

Metamaterials are composed of small structural units in the same manner matter is made up of small units called atoms. The main difference is that these structural units are artificial structures composed of conventional materials. They are artificial periodic structures in which lattice constants are much smaller compared to the wave length of an incident electromagnetic wave. Thus, the material seems to be homogeneous. This distinguishes a metamaterial from frequency-selective surfaces and photonic crystals. ${ }^{203}$ The materials with negative permittivity and permeability are called DNG materials or LHMs. These materials possess a negative refractive index which is not normally found in natural materials. They obey Snell's law, but rays get refracted towards the same side of normal on entering the material. Metamaterials show some more anomalous phenomena like a reverse Doppler effect and backward propagation of the phase front. ${ }^{196}$ That is, for a plane wave incident on the material, energy propagates as in conventional materials, but phase fronts move in an opposite direction. The Doppler effect is observed whenever a relative motion between source and observer occurs. Materials with a negative refractive index show an abnormal frequency shift in comparison to conventional materials as a consequence of relative motion between a wave source and the observer. Therefore, such materials can be engineered according to the desired application for a specific frequency range.

\section{Applications}

Recent advancements in nanotechnology and molecular engineering have helped the development of materials with exotic electromagnetic responses. Intense research on metamaterials and metamaterial-inspired structures has lead to the discovery of advanced applications in microwave to optical frequencies. Some of the applications in the microwave regime are discussed below.

\section{Waveguide Devices}

Sub-wavelength cavities, resonators and ultrathin open waveguides can be implemented using metamaterials. ${ }^{202}$ Mahmoud et al. designed a patch resonator consisting of an outer ring of conventional dielectric material and inner ring of DNG metamaterial. $^{204}$ It causes a field discontinuity at the interface and reduces the size of cavity in its dominant mode. The resonance frequency of the dominant mode in the cavity resonator can be tuned to the desired value by changing the dimensions of the annular ring patch resonators. ENG metamaterials can be used as a waveguide liner, which supports the propagation of electromagnetic waves below the cut-off frequency of an empty rectangular waveguide. $^{205}$

\section{Antenna Applications}

A metamaterial-based leaky wave antenna with tunable radiation angle and beam width has been designed by introducing varactor diodes. The radiation angle is controlled by voltages at fixed 


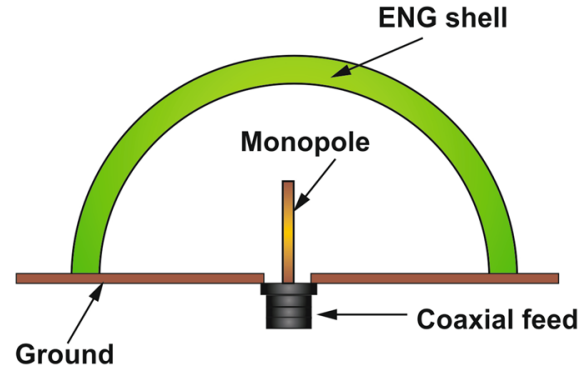

(a)

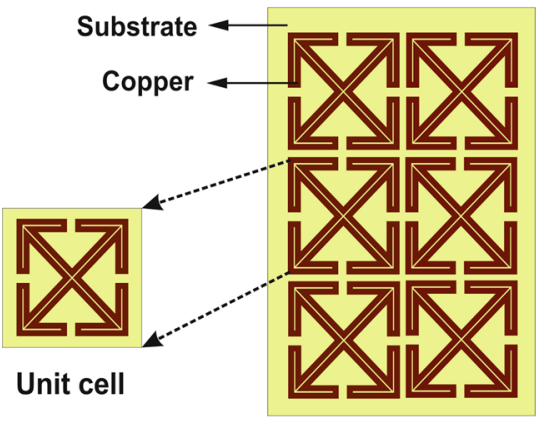

(c)

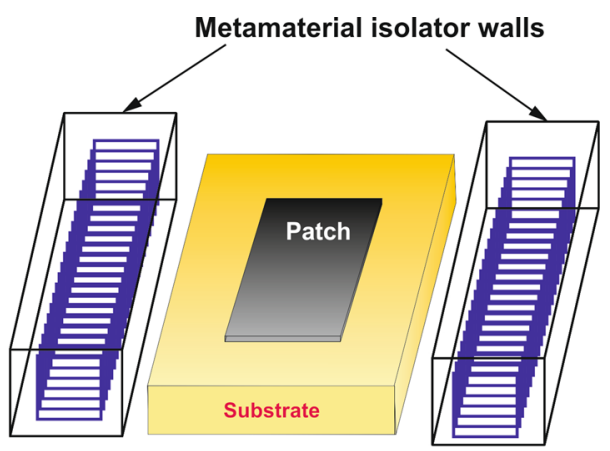

(b)

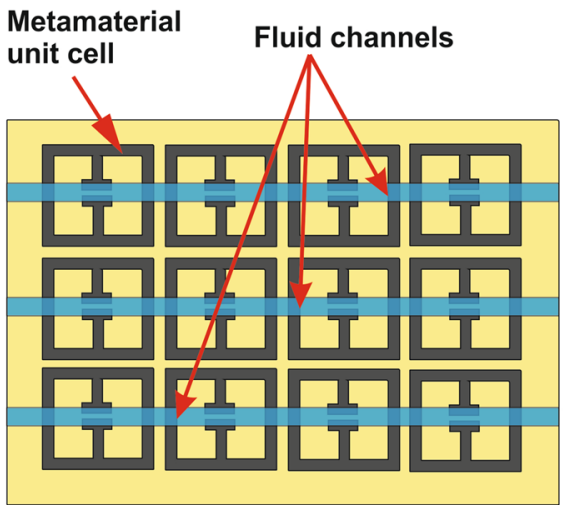

(d)
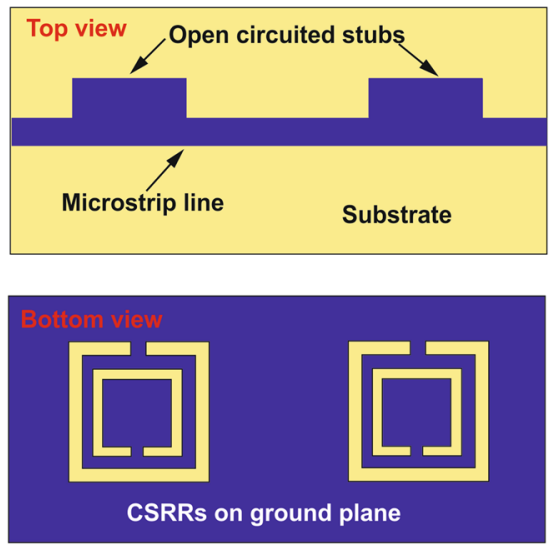

(e)

Fig. 8. Metamaterial-based microwave devices: (a) electrically small antenna using ENG metamaterial (modified and redrawn using the data from Ref. 208), (b) metamaterial isolators for surface wave suppression in a microstrip patch antenna (modified and redrawn using the data from Ref. 212), (c) tetra arrow cave structure metamaterial for EMI/EMC applications (modified and redrawn using the data from Ref. 216), (d) fluid tunable metamaterial film adapted with permission from Ref. 227, copyrighted by the American Physical Society and (e) metamaterial-based UWB filter with notch characteristics (modified and redrawn using the data from Ref. 228).

frequency and beam width via nonuniform biasing of the varactor diodes. ${ }^{206}$ Wang et al. investigated the use of metamaterials with negative reflection phase values as a ground plane for a planar resonant cavity antenna. ${ }^{207}$ It alters the resonance condition which helps in maintaining high directivity with a reduced antenna profile in sub-wavelength values. ${ }^{207}$ Ziolkowski et al. designed an electrically small antenna using ENG metamaterial. ${ }^{208}$ The proposed antenna shown in Fig. 8a consists of an electric dipole surrounded by an ENG metamaterial shell. ${ }^{208}$ The ENG shell behaves as an inductive element which compensates for the capacitive nature of the electric dipole and thus forming a combined resonant system of higher efficiency. It has a fractional bandwidth above the value associated with the $\mathrm{Chu}$ limit for the quality factor. A metamaterial antenna with reduced 
radiation hazards has been discussed in Ref. 209 . The electromagnetic interaction between the antenna and a human head can be reduced by modifying the radiation pattern of the monopole antenna using metamaterial substances like SRRs. The power transmission of a stacked dipole antenna can be enhanced by using an array of SRRs as a cover. ${ }^{210}$ The SRR monolayer cover, having a negative permeability, enhances magnetic resonance strength and thereby increases the radiated power. A metamaterial-based thin planar lens antenna has been proposed for massive multiple-input/multipleoutput (MIMO) system. ${ }^{211}$ The lens is formed by two thin, layered metamaterial surfaces that reduce the insertion and reflection loss.

\section{Electromagnetic Insulators}

Embedded circuit metamaterial with highly efficient band stop rejection characteristics can be placed between radiating elements as shown Fig. 8b in order to obtain better isolation. ${ }^{212}$ Thus, the reduction in mutual coupling between the radiating elements allows more control over element current levels. Hence, a densely packed antenna array with high directivity can be achieved.

\section{Wave Propagation}

Enhancement of wave transmission through a sub-wavelength aperture can be achieved by using metamaterial covers with a very low permittivity at the entrance and exit face of the hole. ${ }^{213}$ The metamaterial cover, which acts a receiving leaky wave antenna, collects incident radiation and, at the exit, it acts as a transmitting antenna. Both antennas are coupled through sub-wavelength aperture and allow more power transmission.

\section{RF Lenses}

A metamaterial lens with a negative permeability has enhanced capability in focusing a magnetic field in the near-field region, which can be utilized to improve the signal-to-noise ratio (SNR) in magnetic resonance imaging system. $^{214}$

\section{EMI or EMC Applications}

Chaurasiya et al. proposed a metamaterial microwave absorber for electromagnetic compatibility (EMC) applications. ${ }^{215}$ The proposed metamaterial is basically double-square ring resonators with four splits at their arms. It shows excellent absorption peaks in the $\mathrm{C}$ band and $\mathrm{Ku}$ band. A dual-band polarization-insensitive metamaterial absorber has been reported to exhibit near-unity absorption, by the proper impedance matching of the structure with free-space impedance. ${ }^{216}$ The schematic of the proposed tetra arrow cave metamaterial is depicted in Fig. 8c. This metamaterial-based microwave absorber could be useful in the mitigation of electromagnetic pollution from RF systems. Magnetic metamaterial with an adjustable frequency dependence of permeability has been studied in Ref. 217, which can be used for shifting the magnetic losses to the desired frequency range. Hence, it finds application in designing electromagnetic absorbers, electromagnetic noise suppressors, etc.

\section{Sensors}

A compact metamaterial-based coplanar spiral resonator antenna with omnidirectional radiation pattern gives equal sensitivity in all directions. Any material placed under the resonator affects resonance frequency, which is suitable for sensor applications. ${ }^{218}$ Quasi-lumped resonators coupled with two tapered transmission lines are very suitable for sensing the concentration of liquid mixtures. It works on the principle of shifting resonance frequency with the change in relative permittivity of the samples, which in turn varies with the concentration of the liquid mixtures. ${ }^{219}$

\section{Electromagnetic Property Control}

The dog bone metamaterials can be used for electromagnetic cloaking by effectively routing incident radiation around a target. ${ }^{220}$ A reconfigurable ultra-thin metasurface for radar cross section (RCS) reduction is discussed in Ref. 221 . The reconfigurability is obtained by changing capacitive loading between unit cells, which in turn controls the reflection phase across the surface and thereby scattering incident fields in any arbitrary direction.

\section{Microwave Absorbers}

Fan et al. proposed an active metamaterial-based microwave absorber having wider band width for incidence angles up to $45^{\circ} .{ }^{222}$ The proposed metamaterial consists of two layers: upper layer containing square metal patches connected by non-Foster loads, and interdigitated capacitors are connected between upper and lower substrates. $\mathrm{Hu}$ et al. compared the microwave absorption properties of a traditional square lattice-based metamaterial absorber and honeycomb lattice-based absorbers. ${ }^{223}$ The honeycomb lattice-based metamaterial was designed on flexible polyimide substrate and it showed better absorption properties even under bending conditions.

\section{Biomedical Applications}

Holloway et al. have developed a metamaterialbased sensor to monitor biological fluids. ${ }^{224}$ The proposed sensor consists of two SRRs coupled to a coplanar wave guide through a microfluidic channel. The resonant frequency of the metamaterial varies in accordance with the change in dielectric properties of the fluid inside the channel. Neha et al. proposed a metamaterial-inspired wide-band wearable microstrip antenna for skin cancer detection. $^{225}$ The resonant frequency of the proposed 
Table II. Materials for EMI applications

\begin{tabular}{|c|c|c|c|c|}
\hline Material & EMI SE (dB) & Frequency $(\mathbf{G H z})$ & Thickness (mm) & Refs. \\
\hline $\begin{array}{l}\text { Carbon black/graphite powder/steel } \\
\text { fiber mixed conductive concrete }\end{array}$ & 65 & $1-5.5$ & - & Ref. 9 \\
\hline $\begin{array}{l}0.026 \text { vol.\% Ferromagnetic wires } / 913 \\
\text { E-glass prepregs }\end{array}$ & 18 & $1-2$ & 0.64 & Ref. 28 \\
\hline $\mathrm{CoNbZr} / \mathrm{Cu}$ laminate & 27 & 0.470 & 0.014 & Ref. 29 \\
\hline PVDF-70 vol. $\%$ graphite & 73 & $8.2-18$ & 1.5 & Ref. 58 \\
\hline PVDF-70 vol.\% graphite & 90 & $8.2-18$ & 2 & Ref. 58 \\
\hline PVDF-30 vol.\% nAg & 45 & $8.2-18$ & 1 & Ref. 58 \\
\hline Polyaniline nanofiber graphite composite & 80 & $8.2-18$ & 1 & Ref. 59 \\
\hline $\begin{array}{l}\text { Polyaniline nanofibers-graphite composite-based } \\
\text { cotton nylon fabrics }\end{array}$ & 15 & $8.2-18$ & 0.1 & Ref. 59 \\
\hline 5 wt.\% Ag 10 wt.\% graphite/PVDF composites & $<25$ & $8-12$ & - & Ref. 108 \\
\hline \multirow[t]{3}{*}{ PVDF/6 wt.\% Ni composite } & 24.7 & $18-26.5$ & 0.3 & Ref. 174 \\
\hline & 15.4 & $18-26.5$ & 0.2 & Ref. 174 \\
\hline & 35.4 & $18-26.5$ & 0.5 & Ref. 174 \\
\hline
\end{tabular}

Table III. Materials for antenna applications and their properties

\begin{tabular}{|c|c|c|}
\hline Applications & Material & Characteristics \\
\hline $\begin{array}{l}\text { Antenna minia- } \\
\text { turization }\end{array}$ & $\begin{array}{c}\text { Porous ferrite ceramics } \mathrm{Ni}_{0.5} \mathrm{Zn}_{0.3} \mathrm{Co}_{0.2} \mathrm{Fe}_{1.98} \mathrm{O}_{4-x}{ }^{32} \\
\text { Magneto-dielectric nanocomposites (cobalt } \\
\text { nanoparticles in fluoropolymer matrix })^{34}\end{array}$ & $\begin{array}{l}\text { Porosity }=50 \% \\
\text { 38.54\% reduction in size compared to an antenna } \\
\text { on FR4 substrate }\end{array}$ \\
\hline $\begin{array}{l}\text { Gain e } \\
\text { men }\end{array}$ & FeCo nanoparticles or thin films ${ }^{35}$ & $\begin{array}{c}\text { Increment in gain } 9 \mathrm{~dB} \text { with external magnetic } \\
\text { field } 10^{-1} \text { Tesla }\end{array}$ \\
\hline $\begin{array}{l}\text { Mutual coupling } \\
\text { control }\end{array}$ & Yttrium iron garnets (YIGs) ${ }^{33}$ & $\begin{array}{c}\mathrm{S}_{21}=-17.2 \mathrm{~dB} \text { at an external applied magnetic } \\
\text { field of } 1600 \text { Oe }\end{array}$ \\
\hline $\begin{array}{l}\text { Frequency } \\
\text { reconfigura- } \\
\text { tion }\end{array}$ & $\begin{array}{l}\mathrm{Co}_{2} \mathrm{Z} \text { hexaferrite }\left(\mathrm{Ba}_{3} \mathrm{Co}_{2} \mathrm{Fe}_{24} \mathrm{O}_{41}\right)^{31} \\
\text { Fe60Co40 nanoparticles or thin thin films }\end{array}$ & $\begin{array}{c}\text { Tuning range } \approx 360-900 \mathrm{MHz} ; \text { external } \\
\text { magnetic field of } 1.755 \mathrm{kOe} \\
26 \% \text { change in permeability; tuning range } 1.9- \\
2 \mathrm{GHz}\end{array}$ \\
\hline $\begin{array}{l}\text { Pattern reconfig- } \\
\text { uration }\end{array}$ & $\begin{array}{l}\text { Multilayered lead zirconate, YIG } \\
\text { and gadolinium gallium garnet } \\
\text { Liquid crystal }^{369}\end{array}$ & $\begin{array}{l}-20^{\circ} \text { to }+20^{\circ} \text { shift in main beam direction with } \\
\text { magnetic field variation } 54.8-55.6 \mathrm{kA} / \mathrm{m} \\
-5^{\circ} \text { to }+9^{\circ} \text { main beam shift for tuning voltage } \\
0-60 \mathrm{~V}\end{array}$ \\
\hline $\begin{array}{l}\text { Polarization } \\
\text { reconfigura- } \\
\text { tion }\end{array}$ & $\begin{array}{l}\text { Liquid crystals }^{106} \\
\text { Yttrium iron garnets }(\mathrm{YIGs})^{192}\end{array}$ & $\begin{array}{c}\text { Dual linear to dual circular polarization tuning } \\
\text { voltage } 0-40 \mathrm{~V} \\
\text { Linear to elliptical polarization with external } \\
\text { magnetic field (5000 Oe) }\end{array}$ \\
\hline $\begin{array}{l}\text { Higher radiation } \\
\text { efficiency }\end{array}$ & Eccostock HiK ${ }^{241}$ & $\begin{array}{c}\text { Radiation efficiency }=96 \% \text { (10\% more than } \\
\text { microstrip patch antenna) }\end{array}$ \\
\hline
\end{tabular}

wearable antenna is sensitive to the dielectric properties of skin tissues. The difference in electrical properties of malignant and normal skin tissues can be utilized to diagnose skin cancer.

\section{Tunable Devices}

A microfluidic switchable metamaterial-based tunable filter has been discussed in Ref. 226. It consists of a metallic resonator structure designed on PDMS substrate backed by a microfluidic channel and metal plate. The absorption peak of the resonator can be tuned by introducing a liquid droplet in the channel. The resonance frequency varies with the change in diameter and dielectric properties of the liquid droplet. Gordon et al. developed a fluid tunable metasurface. ${ }^{227}$ The proposed tunable metasurface shown in Fig. $8 \mathrm{~d}$ is suitable for monitoring chemical processes using microwaves. Microfluidic channels were realized using polymer in the OE2 resonator structure, and the 
Material

NiCoZn spinel ferrites ${ }^{20}$

20 vol.\% Annealed Fe/C core/shell nanoparticles ${ }^{51}$

20 vol.\% Annealed Fe/C core/shell nanoparticles ${ }^{51}$

60 wt. $\%$ MWCNTs/ $/ \mathrm{Fe}^{52}$

60 wt.\% MWCNTs/Co ${ }^{52}$

60 wt. $\%$ MWCNTs/ $\mathrm{Ni}^{52}$

1.3 wt. $\% \mathrm{FeCo} / \mathrm{CNTs}^{53}$

1.3 wt.\% FeCoNi/CNTs ${ }^{53}$

30 wt.\% Fe/helical CNTs ( $\left.{ }^{*} \mathrm{C}-500\right)$ paraffin composites ${ }^{54}$

30 wt.\% Fe/helical CNTs ( ${ }^{*} \mathrm{C}-600$ ) paraffin composites ${ }^{54}$

5 wt.\% Carbon black/50 wt.\% SiC composite ${ }^{56}$

Helical carbon nanofiber-coated carbon fiber composites ${ }^{64}$

Titanium carbonitride $\left(\mathrm{TiN}_{0.2} \mathrm{C}_{0.8}\right)^{66}$

40 wt.\% Silica-Ni-C composite Shelly hollow microspheres ${ }^{67}$

2 wt.\% CNT/rice husk ash composite ${ }^{127}$
20 vol.\% Annealed $\mathrm{Fe} / \mathrm{C}$ core/shell nanoparticles ${ }^{51}$ 40 wt.\% Hexagonal BNNC/graphite nanoflake composites ${ }^{55}$

40 wt.\% Rice husk/nontoxic glue binder ${ }^{139}$

\begin{tabular}{|c|c|}
\hline $\begin{array}{c}\text { Abs. freq. } \\
\text { bandwidth } \\
-10 \mathrm{~dB}^{\text {to } 20 \mathrm{~dB}^{*}}(\mathrm{GHz})\end{array}$ & Thickness (mm) \\
\hline $8.64-11.2 *$ & 3.15 \\
\hline $3.95-5.85$ & 3.2 \\
\hline $8.2-12.4$ & 1.9 \\
\hline $12.4-18$ & 1.4 \\
\hline $2.04-3.47 *$ & $3.36-5.57$ \\
\hline $2.35-3.51 *$ & $4.18-6.82$ \\
\hline $1.83-3.07 *$ & $3.77-6.56$ \\
\hline $7.4-15.4$ & 2 \\
\hline $12.4-18$ & 2 \\
\hline $6.67-9.17 *$ & $1.29-1.46$ \\
\hline $15.83-18 *$ & $2.50-3.03$ \\
\hline $7.41-18.0 *$ & $1.28-2.98$ \\
\hline $9.70-12.98$ & 2 \\
\hline $13.4-17.28$ & 1.5 \\
\hline$\approx 7-13$ & 2 \\
\hline $8.2-18$ & 2.5 \\
\hline $11.1-13.6$ & 1.32 \\
\hline $5.7-18$ & $2.4-4$ \\
\hline $12.48-16.47$ & 2 \\
\hline $6.3-7.1$ & 37.5 \\
\hline
\end{tabular}

(\# pyrolysis temperature, ${ }^{*}-20 \mathrm{~dB}$ absorption frequency bandwidth). transmission response of the metasurface varies with the dielectric properties of the fluid in the gap.

\section{Microwave Filters}

Ali et al. designed an UWB filter with notch characteristics to reduce interference from highperformance local area networks. ${ }^{228}$ The proposed filter is designed using complementary SRR unit cells of negative permittivity over microstrip lines as shown in Fig. 8e. It has bandwidth of $8.4 \mathrm{GHz}$ with notch characteristics at $5.4 \mathrm{GHz}$. Hence, it can be used to reduce interference between UWB systems and high-performance local area networks.

\section{OTHER IMPORTANT MATERIALS}

There are many other classes of materials used in microwave electronics. The demand for materials with superior electromagnetic performance has lead to development of glass ceramics, low-loss ceramic dielectric materials, polymer ceramic composites, high-temperature co-fired ceramics (HTCCs), lowtemperature co-fired ceramics (LTCCs) and ultralow-temperature co-fired ceramics (ULTCCs), etc. There is a huge demand for low-loss microwave substrate materials development of $5 \mathrm{G}$ and millimeter communication systems. The characterization of materials is a prerequisite for different frequency band applications. Therefore, we have to learn more about the properties of materials at higher frequencies, and development of new composite materials is necessary to achieve compact and highly efficient communication systems. Some of the important materials which have not been explained in the previous sections are briefly discussed below.

The research and development of electromagnetic shielding materials have more significance in the present scenario due to the ever-increasing electromagnetic pollution. Electromagnetic interference shielding effectiveness (EMI SE) is a measure of attenuation of impinging electromagnetic waves. Absorption and reflection mechanisms contribute to electromagnetic shielding. Materials with high dielectric or magnetic losses are preferred for shielding applications. Table II depicts various electromagnetic shielding materials and their shielding effectiveness.

Antennas are inevitable devices in wireless communication systems. An ideal substrate for antenna applications should possess low dielectric loss, mechanical and thermal stability and an optimum dielectric constant (i.e., high permittivity for miniaturization and low permittivity for high-speed signal transmission). Some of the mainly used materials and their applications are given in Table III. Microwave absorbers are studied based on their thickness, density and absorption bandwidth. Table IV gives a clear picture of different microwave-absorbing materials that have been discussed in this paper and their absorption characteristics. 


\section{Low-Loss Ceramic Dielectric Materials}

Low-loss dielectric materials are generally characterized by a low loss tangent and low temperature coefficient of resonant frequency. Materials with low permittivity and high quality factor are recommended for millimeter applications due to their low insertion loss, increased signal speed and low electrical noise. The properties and applications of dielectric ceramic materials at microwave frequencies were reported in literature many years back. ${ }^{229}$ Sebastian et al. have reviewed the properties and applications of low-loss dielectric ceramic materials. ${ }^{230}$ Ceramics with a dielectric constant in the range 25-50 are preferred for satellite communications and mobile base stations, whereas high-permittivity ceramics are employed for mobile phone handsets and other circuit component miniaturization. ${ }^{230}$ The emerging millimeter-wave and 5G systems requires substrates with high quality factor, good thermal stability and dielectric permittivity in the range 6-20. Sebastian et al. have proposed possible dielectric materials for millimeter-wave applications such as $\mathrm{Mg}_{2} \mathrm{SiO}_{4-}, \mathrm{ZnAl}_{2} \mathrm{O}_{4} \mathrm{TiO}_{2-}$, $\mathrm{Mg}_{4} \mathrm{Ta}_{2} \mathrm{O}_{9-}$ and $\mathrm{Al}_{2} \mathrm{O}_{3}$-based materials. ${ }^{230}$ Puszkarz et al. synthesized barium titanate dielectric ceramics using titanate nanosheets and barium ions. ${ }^{231}$ The developed barium titanate ceramic exhibits low dielectric loss and dielectric constant of 32 at $1100^{\circ} \mathrm{C}$ which makes it suitable for device miniaturization. $(1-x) \mathrm{ZnAl}_{2} \mathrm{O}_{4-x} \mathrm{TiO}_{2},{ }^{232} \mathrm{LiMgPO}_{4}{ }^{233}$ zinc borosilicate glass- $\mathrm{Al}_{2} \mathrm{O}_{3}$ composites ${ }^{234}$ and $\mathrm{LiMgVO}_{4}{ }^{235}$ are some of the low-loss dielectric ceramics reported in literature as microwave substrates. Sebastian et al. have listed different lowloss dielectric ceramic materials and their properties in Ref. 3.

Richtmyer showed that certain dielectric material can function as electric resonators and their resonance frequency depends on the physical dimension of the material and its permittivity. ${ }^{236} \mathrm{He}$ called such materials dielectric resonators (DRs). DRs play a major role in the miniaturization of oscillators, filters and antennas in cellular communication. Advanced knowledge regarding the properties and resonance mechanism of $\mathrm{DRs}$ has been discussed in Ref. 2. DRs are a promising alternative for conventional metal resonant cavities due to their high Qfactor and smaller size and because they can be easily integrated in microwave integrated circuits (MICs). ${ }^{3}$ They find application in DR antennas (DRAs), ${ }^{237}$ DR filters ${ }^{238}$ and DR oscillators (DROs). ${ }^{239}$ DRAs have higher radiation efficiency and significant bandwidth due to the absence of conductor losses. ${ }^{3}$ Maity et al. have investigated the characteristics of $60^{\circ}-30^{\circ}-90^{\circ}$ triangular DRs (TDRs). ${ }^{240}$ Since they occupy less volume as compared with equilateral TDRs, they can be used in designing compact DRAs, filters and DROs. Guha et al. have done a comparative study on the performance of microstrip resonator antennas (MRAs) and
DRAs with the commonly used feeding techniques. ${ }^{241}$ DRAs have wider bandwidth and higher radiation efficiency in comparison to MRAs. But DRAs have slightly lower gain and less mechanical stability. Mrnka et al. designed and fabricated a $1 \times$ 4 linear DRA array using surface-integrated waveguide technology. ${ }^{242}$ The designed antenna array operated at millimeter-wave frequency and exhibited a gain of $16.3 \mathrm{dBi}$. Chakraborty et al. designed an elliptical DRA operating over the X-band. ${ }^{243} \mathrm{It}$ has a dielectric constant $\left(\varepsilon_{r}\right)$ of 10 and impedance bandwidth of $47 \%$. DRA technology has been reviewed in detail in Refs. 244, 245 and 246.

Cameron et al. have discussed different configurations of DR filters such as single-mode, dual-mode and triple and quadruple modes of operation. ${ }^{247}$ Generally, DR filters consist of DRs arranged in planar or axial configuration inside cavities. An iris provides the necessary coupling between adjacent resonators. $\mathrm{TE}_{01 \delta}(\mathrm{TEH})$ is the dominant mode of operation and single-mode $\mathrm{TE}_{01 \delta}$ filters are widely used in wireless communication. ${ }^{247}$ In comparison with single-mode DR filters, dual-mode and triplemode DR filters show volume reduction of nearly $30 \%$ and $50 \%$, respectively. Wang et al. reviewed the properties and applications of commercially available DRs. ${ }^{248}$ They have also discussed TE-mode, dual-mode and mixed-mode DR filter configurations and their corresponding frequency responses. Zhou et al. designed a DR-based tunable oscillator. ${ }^{239}$ The designed oscillator can be incorporated in microwave integrated circuits using substrate-integrated waveguide technology, and it showed a high Qfactor of 13,000 low-phase noise. DROs have several advantages such as compact size, high-temperature and high-frequency stability, 2increased immunity to noise and high efficiency.

\section{Low-Loss Polymer Ceramic Composites}

Microwave substrates used for high-speed signal transmission and device miniaturization are required to possess low dielectric loss, reasonable relative permittivity, high-frequency compatibility, excellent mechanical and thermal stability and high thermal conductivity to avoid overheating. ${ }^{3}$ Ceramics are known to possess high relative permittivity and excellent thermal properties, but the brittle nature of ceramics limits their application in microwave packaging technology. Polymers exhibit low thermal conductivity, higher coefficient of thermal expansion (CTE) and low processing temperature. Polytetrafluoroethylene (PTFE), polyethylene, polystyrene, high-density polyethylene (HDPE), polyether ether ketone (PEEK), polyamide, LCP and epoxy resin are the commonly used polymers. ${ }^{3}$ The strategy of mixing ceramics in low-loss polymer matrix provides an efficient microwave substrate for microelectronic packaging due to the optimum permittivity, low loss, flexibility and low processing temperature compared to conventional silicates and 
aluminates. ${ }^{249}$ The volume fraction, size, shape, connectivity between phases and dielectric properties of ceramic fillers determine the overall properties of polymer ceramic composites.

There are mainly two types of polymer matrices used in polymer ceramic composites: thermoplastic and thermoset polymers. Thermoplastic polymers have higher chemical inertness and environmental tolerance as compared to thermosets. PTFE is a well-known thermoplastic, whereas polyamide is the commonly used thermoset used in polymer ceramic composites. Sebastian et al. synthesized polyethylene ceramic composites by incorporating ceramic fillers of dielectric constant varying from 10 to 110 in polyethylene polymer matrix and observed that dielectric losses increases with increase in ceramic filler loading. ${ }^{249}$ Subodh et al. developed polyethylene and epoxy-based ceramic composites. ${ }^{250} \mathrm{Sr}_{9} \mathrm{Ce}_{2} \mathrm{Ti}_{12} \mathrm{O}_{36}$ is used as the ceramic filler due to its high dielectric constant and low losses. ${ }^{251}$ Polyethylene ceramic composite $\left(\varepsilon_{r}=12.1\right.$ and $\tan \delta=0.004)$ and epoxy ceramic composite $\left(\varepsilon_{r}=14.1\right.$ and $\left.\tan \delta=0.022\right)$ exhibit low dielectric losses with comparatively high dielectric constant. ${ }^{250}$ They continued studies on other polymer composites materials such as $50 \% \mathrm{Sr}_{2} \mathrm{Ce}_{2} \mathrm{Ti}_{5} \mathrm{O}_{15}-$ filled polystyrene ceramic composites and found them to be a promising microwave substrate for lowcost device miniaturization. Koulouridis et al. prepared polymer ceramic composites by mixing ceramic powder with PDMS. ${ }^{252}$ Since the developed polymer composites are flexible with low dielectric losses, they can be used as a microwave substrate for antennas and filters. The dielectric constant and loss tangent of the composite vary with the volume fraction of ceramic power added in the polymer matrix.

PTFE, commercially known as Teflon, is a potential microwave substrate due to its lower moisture absorption, excellent chemical stability and good electrical and thermal insulating properties. Anjana et al. reported the development of a low-loss PTFE ceramic composite by incorporating $\mathrm{CeO}_{2}$ in the polymer matrix. ${ }^{253}$ Instead of using single material, combination of different materials provides a higher degree of freedom to control electrical, magnetic, thermal and mechanical properties of the material. Thomas et al. reported the development of polymer ceramic composites by incorporating $\mathrm{Sm}_{2} \mathrm{Si}_{2} \mathrm{O}_{7}$ in PTFE matrix. ${ }^{254}$ The proposed composite showed a low dielectric constant, less dielectric loss and excellent thermal conductivity, which makes it useful in packaging and substrate applications. $40 \%$ of silane treated with $\mathrm{Mg}_{2} \mathrm{SiO}_{4}$-filled diglycidyl ether of bisphenol A (DGEBA) composites are reported to possess low $\mathrm{CTE}$ in comparison to untreated $\mathrm{Mg}_{2} \mathrm{SiO}_{4} /$ DGEBA composites. ${ }^{255}$ Silane increases the interface contact between polymer phase and ceramic fillers and reduces CTE while maintaining the same dielectric constant. The composition and dielectric properties of various polymer ceramic composites are reviewed elaborately in Ref. 3.

\section{Glass Ceramics}

Glass has several advantages such as good thermal properties and material homogeneity. But high dielectric loss and limited range of permittivity make them unsuitable for applications at high frequency. Glass ceramics possess the advantages of glass along with low dielectric losses and wide range of permittivity. ${ }^{3}$ Excellent homogeneity and pore-free structure can be obtained by suitable temperature treatment. Thermodynamically, glass is in a metastable state. Appropriate heat treatment is used for the partial crystallization of glass to achieve a pore-free structure, superior thermal properties and desired dielectric properties. The synthesis of glass ceramics involves melting, shaping and nucleation followed by growth of new crystal phases at nucleation sites. Nucleation can be homogeneous or heterogeneous. Nucleation agents such as $\mathrm{TiO}_{2}$ are used to increase the rate of nucleation. ${ }^{256}$ Lo et al. studied the influence of sintering and nucleation agent in the dielectric properties of anorthite/glass ceramics. ${ }^{256} \mathrm{TiO}_{2}$ and $\mathrm{B}_{2} \mathrm{O}_{3}$ are used as nucleation agent and sintering aid, respectively. It is found that $\mathrm{B}_{2} \mathrm{O}_{3}$ improved sintering, making it suitable for low-temperature co-fired ceramics applications. The dielectric constant is found to be decreased with the decrease in $\mathrm{TiO}_{2}$ concentration. Glass ceramics of Ba-Ti-Al-Si-O have been reported as good dielectric materials for antenna miniaturization, ${ }^{257}$ as they exhibit low loss, high permittivity, low CTE and excellent homogeneity. George et al. studied the properties of Lithium Calcium Silicate $\left(\mathrm{Li}_{2} \mathrm{CaSiO}_{4}\right)$ (LCS) ceramics and found that the sintering temperature can be lowered by the addition of borosilicate glasses without affecting the dielectric properties of LCS. $^{258}$ Glass exhibits relatively higher permittivity and CTE compared to ceramics. The addition of glass improves densification and acts a potential sintering agent to lower the sintering temperature of LCS ceramics. It is recommended for study ${ }^{3}$ to obtain advanced knowledge on glasses and glass ceramics used for microwave applications.

\section{Multilayer Ceramics}

Advanced packaging technology demands compact and cost-effective high-frequency compatible multilayer packaging. Individual layers are developed by mixing ceramics, binding agents and glass powders. Glass or oxides having low melting points are usually employed for lowering the sintering temperature of ceramics. ${ }^{3}$ Planar or vertical electrical feedthroughs are created depending on specific applications and they are filled with conductive paste. This is followed by the stacking of different layers and sintering. Based on sintering 

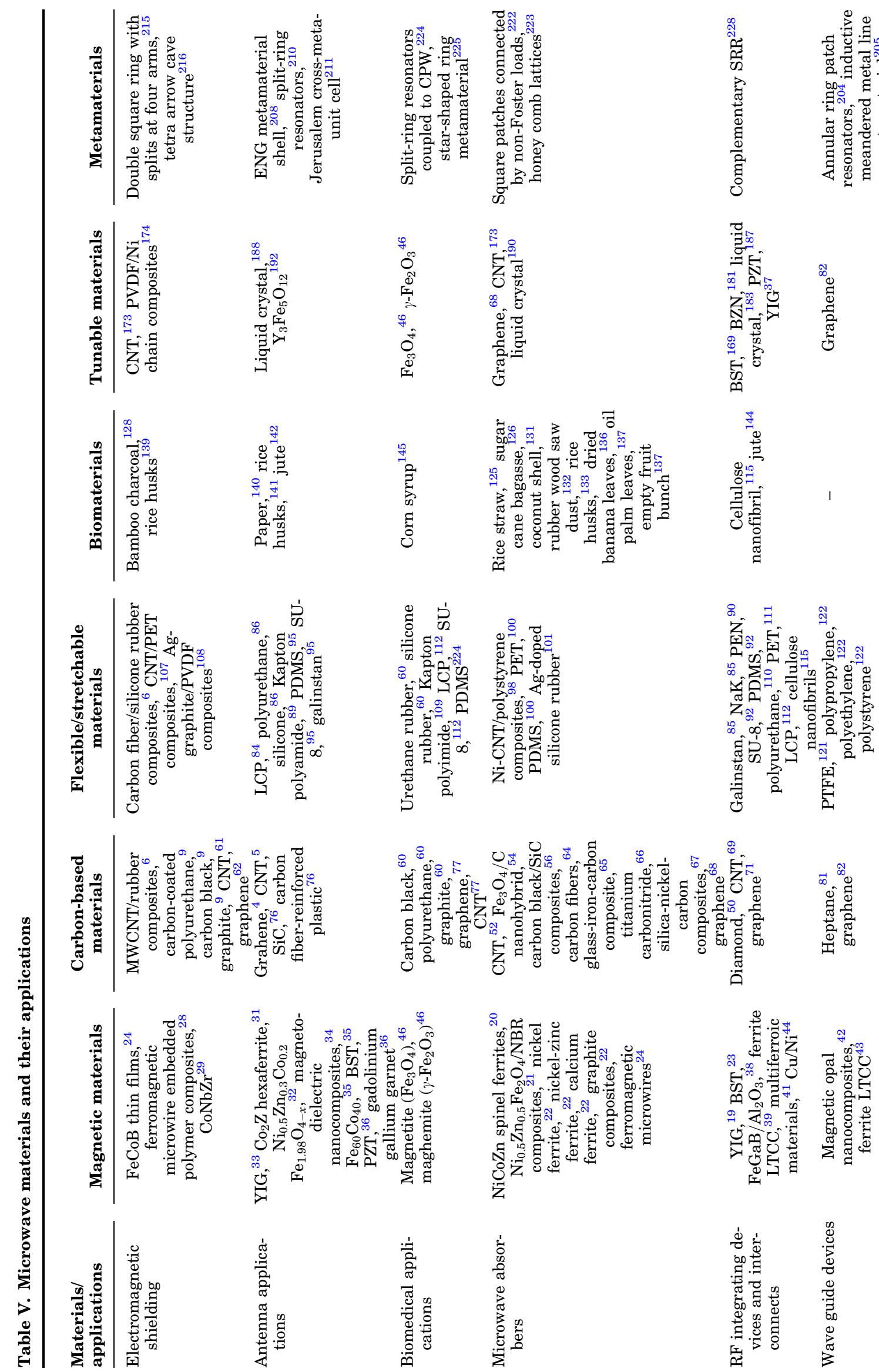
temperature, multilayer ceramics can be classified into HTCCs, LTCCs and ULTCCs). ${ }^{3}$ LTCCs are cofired within the temperature range $700-950^{\circ} \mathrm{C}$, whereas HTCCs are co-fired at temperature more than $1500^{\circ} \mathrm{C}$. The multilayer ceramics with a sintering temperature lower than $700^{\circ} \mathrm{C}$ are considered as ULTCCs. LTCC with low permittivity and low dielectric losses finds application in millimeterwave communications. Gold and silver are not recommended for HTCC applications because they will melt and flow down at such higher temperature. As such, they are used in LTCC technology, whereas tungsten and manganese are used in HTCC applications. $^{3}$

Vahdati et el. designed a transmitter module containing a millimeter-wave phase antenna array integrated on LTCC material which showed high gain and received $-37.3 \mathrm{dBm}$ power at a distance of $1 \mathrm{~m} .{ }^{259}$ Titz et al. reported a millimeter-wave module comprising transmitting and receiving antennas integrated on HTCC substrate that showed an acceptable performance level. ${ }^{260}$ LTCC materials with low permittivity and low dielectric losses find tremendous applications in high-speed communication devices. Zhou et al. have studied the dielectric properties of $\mathrm{NaAgMoO}_{4}$ material for ultra-lowtemperature co-fired ceramics applications. ${ }^{261}$ The proposed material is found to be chemically compatible with silver and aluminum and has low sintering temperature of $400^{\circ} \mathrm{C}$.

Table $\mathrm{V}$ depicts different categories of materials and their applications at microwave frequencies. Different applications presented in this table are antenna applications, biomedical applications, sensors, tunable devices, electromagnetic shielding applications, etc.

\section{CONCLUSION}

An overview of different materials used in microwave electronics and their applications has been presented in this review. This review gives an overall understanding of various classes of materials used for microwave applications. Among the numerous categories of microwave materials, the seven major categories have been reviewed. It includes magnetic materials, carbon-based materials, flexible materials, biomaterials, tunable materials, phantoms and metamaterials. The other important microwave materials such as low-loss ceramic dielectric materials, low-loss polymer ceramic composites, glass ceramics and multilayer ceramics are discussed in the "Other Important Materials" section. The different classes of microwave materials and their applications are presented in Table I. This study can assist material scientists and microwave researchers in appropriate material selection depending on the diverse applications. On other hand, it also helps in finding the areas which require more attention and advancement in research. 


\section{ACKNOWLEDGMENTS}

This work was financially supported by the University Grants Commission (UGC), Government of India under the Faculty Recharge Program. The authors would like to thank Dr. Sriram K and Mr. Manikandan $\mathrm{N}$ for providing fruitful discussion.

\section{REFERENCES}

1. J. Varghese, N. Joseph, H. Jantunen, S.K. Behera, H.T. Kim, and M.T. Sebastian, Advanced Ceramics and Composites for Defense, Security, Aerospace and Energy Applications (Springer, Berlin, 2018).

2. M.T. Sebastian, Dielectric Materials for Wireless Communication (Elsevier, Amsterdam, 2010).

3. M. Sebastian, R. Ubic, and H. Jantunen, Microwave Materials and Applications (Wiley, Hoboken, 2017).

4. Y. Wu, M. Qu, L. Jiao, Y. Liu, and Z. Ghassemlooy, AIP Adv., 6, 065308 (2016).

5. E. Amram, D. Senic, L.W. Taylor, D.E. Tsentalovich, P. Chen, C.L. Holloway, A. Babakhani, C.J. Long, D.R. Novotny, and J.C. Booth, Appl. Phys. Lett., 111, 163109 (2017).

6. J.V. Vas and M.J. Thomas, IEEE Electromang. Compat, 5, 77 (2016).

7. F. Qin and C. Brosseau, J. Appl. Phys., 111, 4 (2012).

8. F. Meng, H. Wang, F. Huang, Y. Guo, Z. Wang, D. Hui, and Z. Zhou, Compos. B Eng., 137, 260 (2018).

9. T. Khalid, L. Albasha, N. Qaddoumi, and S. Yehia, IEEE Trans. Antennas Propag., 65, 2428 (2017).

10. L.P. Carignan, A. Yelon, D. Menard, and C. Caloz, IEEE Trans. Microw. Theory, 59, 2568 (2011).

11. Y.H. Jung, H. Zhang, S.J. Cho, and Z. Ma, IEEE Trans. Electron Devices, 64, 1881 (2017).

12. V.K. Guna, G. Murugesan, B.H. Basavarajaiah, M. Ilangovan, S. Olivera, V. Krishna, and N. Reddy, IEEE Trans. Electron Devices, 63, 4893 (2016).

13. A. Ahmed, I.A. Goldthorpe, and A.K. Khandani, Appl. Phys. Rev., 2, 011302 (2015).

14. S. Geetha, K. Satheesh, C.R. Rao, M. Vijayan, and D. Trivedi, J. Appl. Polym. Sci., 112, 2073 (2009).

15. V.E. Demidov, S. Urazhdin, H. Ulrichs, V. Tiberkevich, A. Slavin, D. Baither, G. Schmitz, and S.O. Demokritov, Nat. Mater., 11, 1028 (2012).

16. V.G. Harris, A. Geiler, Y. Chen, S.D. Yoon, M. Wu, A. Yang, Z. Chen, P. He, P.V. Parimi, and X. Zuo, J. Magn. Magn. Mater., 321, 2035 (2009).

17. R.H. Knerr, IEEE Trans. Microw. Theory, 23, 818 (1975).

18. R.L. Carter, J.M. Owens, and D.K. De, IEEE Trans. Microw. Theory, 32, 1671 (1984).

19. H. How, W. Hu, C. Vittoria, L.C. Kempel, and K.D. Trott, J. Appl. Phys., 85, 4853 (1999).

20. J. Xie, M. Han, L. Chen, R. Kuang, and L. Deng, J. Magn. Magn. Mater., 314, 37 (2007).

21. L. Saini, M.K. Patra, R.K. Jani, G.K. Gupta, A. Dixit, and S.R. Vadera, Sci. Rep. UK, 7, 44457 (2017).

22. V. Mishra, S. Puthucheri, and D. Singh, IEEE Trans. Magn., 53, 1 (2017).

23. N. Setter, D. Damjanovic, L. Eng, G. Fox, S. Gevorgian, S. Hong, A. Kingon, H. Kohlstedt, N. Park, and G. Stephenson, J. Appl. Phys., 100, 051606 (2006).

24. J. Qiu, Y. Wen, P. Li, J. Yang, and W. Li, J. Appl. Phys., 115, 17A304 (2014).

25. L. Liu, L. Kong, G. Lin, S. Matitsine, and C. Deng, IEEE Trans. Magn., 44, 3119 (2008).

26. L.A. Makarova, V.V. Rodionova, Y.A. Alekhina, T.S. Rusakova, A.S. Omelyanchik, and N.S. Perov, IEEE Trans. Magn., 53, 1 (2017).

27. S.D. Sloetjes, H.H. Urdahl, J.K. Grepstad, and E. Folven, AIP Adv., 7, 056325 (2017).
28. F. Qin, H. Peng, N. Pankratov, M. Phan, L. Panina, M. Ipatov, V. Zhukova, A. Zhukov, and J. Gonzalez, J. Appl. Phys., 108, 044510 (2010).

29. K. Yamada, M. Ishida, S. Yutaka, and M. Yamaguchi, in IEEE International Symposium on Electromagnetic Compatibility (EMC) (2011), pp. 432-437.

30. E. Andreou, T. Zervos, F. Lazarakis, A. Alexandridis, K. Dangakis, E. Varouti, G. Fikioris, and J. Vardaxoglou, in Antennas and Propagation Conference (LAPC) (2014), pp. 376-380.

31. Z. Chen, J. Yu, X. Chen, C. Parini, X. Wang, H. Lin, Z. Zhou, T. Nan, and N.X. Sun, in IEEE International Symposium on Antennas and Propagation and USNC/URSI National Radio Science Meeting (2015), pp. 2287-2288.

32. E. Le Guen, A.C. Tarot, and J.L. Mattei, in European Conference on Antennas and Propagation (EuCAP) (2015), pp. 1-5.

33. E. Andreou, T. Zervos, A. Alexandridis, F. Lazarakis, and G. Fikioris, in European Conference on Antennas and Propagation (EuCAP) (2015), pp. 1-5.

34. K. Han, M. Swaminathan, R. Pulugurtha, H. Sharma, R. Tummala, S. Yang, and V. Nair, IEEE Antennas Wirel. Propag., 15, 72 (2016).

35. Y. Malallah, K. Alhassoon, D. Venkatesh, A. Daryoush, C. Chinnasamy, M. Marinescu, and H. Gundel, in IEEE International Symposium on Antennas and Propagation (APSURSI) (2016), pp. 567-568.

36. A. Tatarenko, R. Petrov, A. Nikitin, M. Bichurin, S. Tomita, and T. Ueda, in Progress in Electromagnetic Research Symposium (PIERS) (2016), pp. 2769-2773.

37. Z. Zhang, J. Liu, H. Ding, Z. Feng, and Y. Nie, IEEE Magn. Lett., 8, 1 (2017).

38. Y. Gao, S.Z. Zardareh, X. Yang, T.X. Nan, Z.Y. Zhou, M. Onabajo, M. Liu, A. Aronow, K. Mahalingam, and B.M. Howe, IEEE Trans. Electron Devices, 61, 1470 (2014).

39. S. Yang, D. Vincent, J.R. Bray, and L. Roy, in European Microwave Integrated Circuit Conference (2014), pp. 596599.

40. S. Yang, D. Vincent, J.R. Bray, and L. Roy, IEEE Trans. Compon. Packag. Manuf., 5, 879 (2015).

41. H. Lin, Y. Gao, X. Wang, T. Nan, M. Liu, J. Lou, G. Yang, Z. Zhou, X. Yang, and J. Wu, IEEE Trans. Magn., 52, 1 (2016).

42. G. Makeeva, O. Golovanov, A. Rinkevich, and M. Samoylovich, in European Microwave Conference (EuMC) (2014), pp. $1162-1165$.

43. A. Nafe and A. Shamim, IEEE Trans. Microw. Theory, 63, 2264 (2015).

44. A. Rahimi and Y.K. Yoon, IEEE Microw. Wirel. Compon., 26, 258 (2016)

45. Y. Chen, X. Fan, Y. Xie, Y. Zhou, T. Wang, J.D. Wilson, R.N. Simons, S.T. Chui, and J.Q. Xiao, in IEEE MTT-S International Microwave Symposium (2015), pp. 1-4.

46. K. Kim, T. Seo, K. Sim, and Y. Kwon, IEEE Trans. Microw. Theory, 64, 2184 (2016).

47. A. Kaur, X. Yang, and P. Chahal, IEEE Trans. Compon Packag. Manuf., 6, 1766 (2016).

48. J.S. Moon and D.K. Gaskill, IEEE Trans. Microw. Theory, 59, 2702 (2011).

49. G. Deligeorgis, M. Dragoman, D. Neculoiu, D. Dragoman, G. Konstantinidis, A. Cismaru, and R. Plana, Appl. Phys. Lett., 95, 073107 (2009).

50. S. Russell, S. Sharabi, A. Tallaire, and D.A. Moran, IEEE Trans. Electron Devices, 62, 751 (2015).

51. O. Khani, M.Z. Shoushtari, and M. Farbod, Phys. B., 477, $33(2015)$

52. F. Wen, F. Zhang, and Z. Liu, J. Phys. Chem. C, 115, 14025 (2011).

53. R. Lv, F. Kang, J. Gu, X. Gui, J. Wei, K. Wang, and D. Wu, Appl. Phys. Lett., 93, 223105 (2008).

54. X. Qi, Q. Hu, J. Xu, R. Xie, Z. Bai, Y. Jiang, S. Qin, W. Zhong, and Y. Du, Mater. Sci. Eng. B-Adv., 211, 53 (2016)

55. B. Zhong, W. Liu, Y. Yu, L. Xia, J. Zhang, Z. Chai, and G. Wen, Appl. Surf. Sci., 420, 858 (2017). 
56. X. Liu, Z. Zhang, and Y. Wu, Compos. B Eng., 42, 326 (2011).

57. J. Chameswary, K. Jithesh, S. George, S. Raman, P. Mohanan, and M. Sebastian, Mater. Lett., 64, 743 (2010).

58. N. Joseph, J. Varghese, and M.T. Sebastian, Compos. B Eng., 123, 271 (2017).

59. N. Joseph, J. Varghese, and M.T. Sebastian, Polym. J., 49, 391 (2017)

60. J. Garrett and E. Fear, IEEE Antennas Wirel. Propag., 13, 599 (2014).

61. E. Decrossas, M.A. El Sabbagh, V.F. Hanna, and S.M. ElGhazaly, in IEEE International Symposium on Electromagnetic Compatibility (2012), pp. 428-433.

62. T. Zhang, B. Zhong, L. Xia, and G.Wen, Mater. Chem. Phys., 170, 303 (2016).

63. A. Kolanowska, D. Janas, A.P. Herman, R.G. Jedrysiak, T. Gizewski, and S. Boncel, Carbon, 126, 31 (2018).

64. L. Liu, P. He, K. Zhou, and T. Chen, AIP Adv., 3, 082112 (2013).

65. Z. An and J. Zhang, J. Mater. Chem. C, 4, 7979 (2016).

66. X. Hong, Q. Wang, Z. Tang, W. Khan, D. Zhou, and T. Feng, J. Phys. Chem. C, 120, 148 (2015).

67. Z. An and J. Zhang, Dalton T., 45, 2881 (2016).

68. D. Yi, X.C. Wei, and Y.L. Xu, IEEE Trans. Microw. Theory, 65,2819 (2017).

69. J.M. Bethoux, H. Happy, G. Dambrine, V. Derycke, M. Goffman, and J.P. Bourgoin, IEEE Electr. Device Lett., 27, 681 (2006).

70. S. Farhana, A.Z. Alam, and S. Khan, in IEEE Regional Symposium on Micro and Nanoelectronics (2015), pp. 1-4

71. L. Liao, Y.C. Lin, M. Bao, R. Cheng, J. Bai, Y. Liu, Y. Qu, K.L. Wang, Y. Huang, and X. Duan, Nature, 467, 305 (2010).

72. M. Aldrigo, M. Dragoman, S. Xavier, and A. Ziaei, in International Semiconductor Conference (CAS) (2016), pp. 63-66.

73. A. Mehdipour, I.D. Rosca, A.R. Sebak, C.W. Trueman, and S.V. Hoa, IEEE Trans. Antennas Propag., 59, 3572 (2011).

74. M. Dragoman, D. Neculoiu, A.C. Bunea, G. Deligeorgis, M. Aldrigo, D. Vasilache, A. Dinescu, G. Konstantinidis, D. Mencarelli, and L. Pierantoni, Appl. Phys. Lett., 106, 153101 (2015)

75. P. Kopyt, B. Salski, M. Olszewska-Placha, D. Janczak, M. Sloma, T. Kurkus, M. Jakubowska, and W. Gwarek, IEEE Trans. Antennas Propag., 64, 2862 (2016).

76. H. Wang, X. Dong, M. Yi, F. Xue, Y. Liu, and G. Liu, IEEE Trans. Antennas Propag., 65, 4443 (2017).

77. S.W. Lan, M.H. Weng, R.Y. Yang, S.J. Chang, Y.S. Chung, T.C. Yu, and C.S. Wu, Materials, 9, 559 (2016).

78. M. Jamlos and A. Ismail, in IEEE International RF and Microwave Conference (2015), pp. 248-251.

79. J. Liu and B. Li, in IEEE MTT-S International Microwave Workshop Series on Advanced Materials and Processes for RF and THz Applications (2015), pp. 1-3.

80. P. Bahoumina, H. Hallil, J.L. Lachaud, D. Rebiere, C. Dejous, A. Abdelghani, K. Frigui, S. Bila, D. Baillargeat, and Q. Zhang, in Symposium on Design, Test, Integration and Packaging of MEMS / MOEMS (2017), pp. 1-4.

81. V. Kumar, A. Vohra, and V. Srivastava, Indian J. Radio Space, 36, 345 (2007).

82. L. Pierantoni, D. Mencarelli, M. Bozzi, R. Moro, S. Moscato, L. Perregrini, F. Micciulla, A. Cataldo, and S. Bellucci, IEEE Trans. Microw. Theory, 63, 2491 (2015).

83. P. Chen and G. Zhang, Sci. China Phys. Mech., 56, 207 (2013).

84. G. DeJean, R. Bairavasubramanian, D. Thompson, G. Ponchak, M. Tentzeris, and J. Papapolymerou, IEEE Antennas Wirel. Propag., 4, 22 (2005).

85. Z. Wu, K. Hjort, and S.H. Jeong, in Proceedings of IEEE (2015), pp. 1211-1225.

86. A. Arriola, J. Sancho, S. Brebels, M. Gonzalez, and W. De Raedt, IET Microw. Antenna Propag., 5, 852 (2011).

87. S.J. Cho, Y.H. Jung, and Z. Ma, IEEE J. Electron Devices, 3, 435 (2015).
88. M. Sharma, S. Sharma, J. Abraham, S. Thomas, G. Madras, and S. Bose, Mater. Res. Express, 1, 035003 (2014).

89. H.R. Khaleel, H.M. Al-Rizzo, D.G. Rucker, and S. Mohan, IEEE Antennas Wirel. Propag., 11, 564 (2012).

90. N. Petrone, I. Meric, T. Chari, K.L. Shepard, and J. Hone, EEE J. Electron Devices, 3, 44 (2015).

91. N. Tiercelin, P. Coquet, R. Sauleau, V. Senez, and H. Fujita, J. Micromech. Microeng., 16, 2389 (2006).

92. T.Y. Chao, C.W. Liang, Y. Cheng, and C.N. Kuo, IEEE Trans. Electron Devices, 58, 906 (2011).

93. R. Alrawashdeh, Y. Huang, and P. Cao, Electron. Lett., 49, 1515 (2013).

94. M. Rizwan, M. Khan, H. He, J. Virkki, L. Sydanheimo, and L. Ukkonen, Electron. Lett., 53, 1054 (2017).

95. S. Cheng, Z. Wu, P. Hallbjorner, K. Hjort, and A. Rydberg, IEEE Trans. Antennas Propag., 57, 3765 (2009).

96. M. Sebastian and L. Namitha, Microwave Materials and Applications, vol. 1, (Wiley, Hoboken, 2017), pp. 537-566.

97. C.Y. Chen, N.W. Pu, Y.M. Liu, L.H. Chen, C.H. Wu, T.Y. Cheng, M.H. Lin, M.D. Ger, Y.J. Gong, and Y.Y. Peng, Compos. B Eng., 135,119 (2018).

98. R. Kumar, T. Narayanan, A. Reena, M. Anantharaman, A. Srivastava, R. Vajtai, and P.M. Ajayan, Appl. Phys. Lett. 99, 113116 (2011).

99. H.M. Lee, Prog. Electromagn. Res., 46, 73 (2014).

100. T. Jang, H. Youn, Y.J. Shin, and L.J. Guo, ACS Photonics, 1, 279 (2014)

101. F. Zhang, Z. Liu, K. Qiu, W. Zhang, C. Wu, and S. Feng, Appl. Phys. Lett., 106, 061906 (2015).

102. F. Zhang, S. Feng, K. Qiu, Z. Liu, Y. Fan, W. Zhang, Q. Zhao, and J. Zhou, Appl. Phys. Lett., 106, 091907 (2015).

103. S. Nikolaou, G.E. Ponchak, J. Papapolymerou, and M.M. Tentzeris, IEEE Trans. Antennas Propag., 54, 1663 (2006).

104. I. Locher, M. Klemm, T. Kirstein, and G. Trster, IEEE Trans. Adv. Packag., 29, 777 (2006).

105. R.A. Liyakath, A. Takshi, and G. Mumcu, IEEE Antennas Wirel. Propag., 12, 603 (2013).

106. O.H. Karabey, S. Bildik, S. Bausch, S. Strunck, A. Gaebler, and R. Jakoby, IEEE Trans. Antennas Propag., 61, 70 (2013).

107. M. Seo, J. Yim, Y. Ahn, F. Rotermund, D. Kim, S. Lee, and H. Lim, Appl. Phys. Lett., 93, 231905 (2008).

108. R. Kumaran, M. Alagar, S. Dinesh, V. Subramanian, and K. Dinakaran, Appl. Phys. Lett., 107, 113107 (2015).

109. H. Bahrami, S.A. Mirbozorgi, R. Ameli, L.A. Rusch, and B. Gosselin, IEEE Trans. Biomed. Circuits Syst., 10, 38 (2016).

110. Y. Sun, E. Menard, J.A. Rogers, H.S. Kim, S. Kim, G. Chen, I. Adesida, R. Dettmer, R. Cortez, and A. Tewksbury, Appl. Phys. Lett., 88, 183509 (2006).

111. L. Sun, G. Qin, H. Huang, H. Zhou, N. Behdad, W. Zhou, and Z. Ma, Appl. Phys. Lett., 96, 013509 (2010).

112. G.T. Hwang, D. Im, S.E. Lee, J. Lee, M. Koo, S.Y. Park, S. Kim, K. Yang, S.J. Kim, and K. Lee, ACS Nano,7, 4545 (2013).

113. D.M. Sun, C. Liu, W.C. Ren, and H.M. Cheng, Small, 9, 1188 (2013).

114. Z. Ma, Y.H. Jung, J.H. Seo, T.H. Chang, S.J. Cho, J. Lee, $\mathrm{H}$. Zhang, and W. Zhou, in IEEE International Electron Devices Meeting (2015), pp. 19.2.1-19.2.4.

115. Y.H. Jung, T.H. Chang, H. Zhang, C. Yao, Q. Zheng, V.W Yang, H. Mi, M. Kim, S.J. Cho, and D.W. Park, Nat. Commun., 6, 7170 (2015)

116. B. Gao, M.M. Yuen, and T.T. Ye, Sci. 7, 45108 (2017).

117. M.I. Khan and F.A. Tahir, Sci. 7, 16059 (2017).

118. K. Grenier, D. Dubuc, P.E. Poleni, M. Kumemura, H. Toshiyoshi, T. Fujii, and H. Fujita, in IEEE Radio and Wireless Symposium (2010), pp. 523-526.

119. O. Korostynska, A. Mason, and A.I. Al-Shamma'a, J. Electromagnet. Wave., 27, 2075 (2013).

120. M. D'Asaro, D. Sheen, and J. Lang, in IEEE Sensors (2016), pp. 1-3.

121. W.M. Bruno and W.B. Bridges, IEEE Trans. Microw. Theory, 36, 882 (1988). 
122. K.Y. Kim, J.R. Sohn, H.S. Tae, and J.H. Lee, in IEEE Antennas and Propagation Society International Symposium (2001), pp. 274-277.

123. H. Yousef, S. Cheng, and H. Kratz, J. Microelectromech. Syst., 18, 154 (2009).

124. A. Bedoya-Pinto, M. Donolato, M. Gobbi, L.E. Hueso, and P. Vavassori, Appl. Phys. Lett., 104, 062412 (2014).

125. H. Nornikman, F. Malek, P. Soh, and A.H. Azremi, in Antennas and Propagation Conference (LAPC) (2010), pp. 313-316.

126. Z. Liyana, F. Malek, H. Nornikman, N.M. Affendi, L. Mohamed, N. Saudin, and A. Ali, in IEEE Symposium on Wireless Technology and Applications (ISWTA) (2012), pp. 66-70.

127. L.Y. Seng, F. Malek, F. Wee, E. Cheng, W. Liu, Z. Liyana, H. Rahim, and A. Ezanuddin, in International Workshop on Electromagnetics: Applications and Student Innovation Competition (2015), pp. 1-4.

128. K. Wu, T. Ting, C. Liu, C. Yang, and J. Hsu. Compos. Sci. Technol., 68, 132 (2008).

129. S. Ozah, J. Gogoi, and N. Bhattacharyya, in Applied Electromagnetics Conference (AEMC) (2009), pp. 1-4.

130. J. Gogoi, S. Sarmah, D. Sarmah, and N. Bhattacharyya, in Applied Electromagnetics Conference (AEMC) (2009), pp. $1-4$.

131. M. Salleh, M. Yahya, Z. Awang, W. Muhamad, A. Mozi, and N. Yaacob, in IEEE Region 10 Conference TENCON (2011), pp.1110-1113.

132. S.M. Se, A. Shaaban, and I.M. Ibrahim, in IEEE Symposium on Wireless Technology and Applications (ISWTA) (2011), pp. 192-197.

133. H. Nornikman, P.J. Soh, F. Malek, A.A.H. Azremi, F. Wee, and R. Ahmad, in Asia-Pacific Symposium on Electromagnetic Compatibility (APEMC) (2010), pp. 916919.

134. H. Nornikman, M.F.B.A. Malek, M. Ahmed, F.H. Wee, P.J. Soh, A.A.A.H. Azremi, S. Ghani, A. Hasnain, and M. Taib, Prog. Electromagn. Res., 111, 141 (2011).

135. F. Malek, M. Mezan, N.M. Affendi, N. Saudin, H. Nornikman, M. Mohamad, L. Mohamed, A. Ali, and K. Juni, in IEEE Asia-Pacific Conference on Applied Electromagnetics (2012), pp. 148-152.

136. Z. Farhany, F. Malek, H. Nornikman, N.M. Affendi, L. Mohamed, N. Saudin, and A. Ali, in IEEE Symposium on Wireless Technology and Applications (2012), pp. 6065 .

137. E. Baharudin, A. Ismail, A. Alhawari, E. Zainudin, D.A.A. Majid, F.C. Seman, and N. Khamis, in IEEE Regional Symposium on Micro and Nanoelectronics (2013), pp. 8588.

138. C. Jin, Q. Yao, J. Li, B. Fan, and Q. Sun, Mater. Des., 85, 205 (2015).

139. M. Iqbal, F. Malek, Y. Lee, L. Zahid, N. Yusof, and F. Abdullah, in IEEE International RF and Microwave Conference (2013), pp. 429-432.

140. G. Shaker, S. Safavi-Naeini, N. Sangary, and M.M. Tentzeris, IEEE Antennas Wirel. Propag., 10, 111 (2011).

141. M.H. Ullah, W. Mahadi, and T. Latef, Sci. 5, 12868 (2015).

142. I. Sfar and L. Osman, in IEEE 15th Mediterranean Microwave Symposium (2015), p. 1.

143. J.H. Seo, T.H. Chang, J. Lee, R. Sabo, W. Zhou, Z. Cai, S. Gong, and Z. Ma, Appl. Phys. Lett., 106, 262101 (2015).

144. K. Zeouga, L. Osman, A. Gharsallah, S. Sankaralingam, and B. Gupta, in IEEE 15th Mediterranean Microwave Symposium (2015), pp. 1-3.

145. G. Bindu, L. Anil, T. Vinu, C.K. Aanandan, and K. Mathew, Prog. Electromagn. Res., 59, 175 (2006).

146. K. Ito, in International Conference on Electromagnetics in Advanced Applications (2016), pp. 600-601.

147. http://niremf.ifac.cnr.it/tissprop/.

148. B. Hattenhorst, M. Mallach, C. Baer, T. Musch, J. Barowski, and I. Rolfes, in IEEE MTT-S International Microwave Bio Conference (2017), pp. 1-4.
149. P. Hall and Y. Hao, Antennas and Propagation for BodyCentric Wireless Communications (Artech House, Norwood, 2006), pp. 18-29.

150. A.T. Mobashsher and A.M. Abbosh, IEEE Microw. Mag., 16,42 (2015).

151. M.S.M. Said and N. Seman, IEEE Trans. Dielect. Electr. Insul., 24, 528 (2017).

152. T. Kobayashi, T. Nojima, K. Yamada, and S. Uebayashi, IEEE Trans. Microw. Theory, 41, 136 (1993).

153. Y. Nikawa, M. Chino, and K. Kikuchi, IEEE Trans. Microw. Theory, 44, 1949 (1996).

154. T. Hikage, Y. Sakaguchi, T. Nojima, and Y. Koyamashita, in IEEE International Symposium on Electromagnetic Compatibility (2007), pp. 1-4.

155. M. Ostadrahimi, R. Reopelle, S. Noghanian, S. Pistorius, A. Vahedi, and F. Safari, in Annual International Conference of the IEEE Engineering in Medicine and Biology Society (2009), pp. 2727-2730.

156. N. Joachimowicz, C. Conessa, T. Henriksson, and B. Duchene, IEEE Antennas Wirel. Propag., 13, 1333 (2014).

157. R. Augustine, S. Raman, and A. Rydberg, in IEEE Conference on Antenna Measurements and Applications (2014), pp. 1-2.

158. B.J. Mohammed and A.M. Abbosh, Microw. Opt. Technol. Lett., 56, 979 (2014).

159. K. Ito, M. Takahashi, and K. Saito, IEICE Trans. Commun., 99, 9 (2016).

160. A.J. Chee, C.K. Ho, B.Y. Yiu, and C. Alfred, IEEE Trans. Ultrason. Ferroelectr., 63, 1852 (2016).

161. N. Joachimowicz, B. Duchene, C. Conessa, and O. Meyer, in European Conference on Antennas and Propagation (EUCAP) (2017), pp. 2719-2722.

162. J. Moll, D. Wcortge, V. Krozer, A. Santorelli, M. Popovic, B. Bazrafshan, F. Hubner, T.J. Vogl, and N. Nikolova, in European Conference on Antennas and Propagation (EUCAP) (2017), pp. 2723-2727.

163. M. Miyakawa and S. Hoshina, in IEEE MTT-S International Microwave Symposium Digest (1996), pp. 10891092.

164. M. Miyakawa and S. Hoshina, in IEEE International Symposium on Electromagnetic Compatibility (2002), pp. 671-676.

165. K. Fukunaga, S. Watanabe, and Y. Yamanaka, Proceedings of EMC'04 SENDAI (2004), pp. 805-808.

166. Y. Okano, K. Ito, I. Ida, and M. Takahashi, IEEE Trans. Microw. Theory 48, 2094 (2000).

167. J.M. Felicio, C.A. Fernandes, and J.R. Costa, in IEEE International Symposium on Antennas and Propagation (APSURSI) (2016), pp. 1049-1050.

168. R. De Paolis, F. Coccetti, S. Payan, A. Rousseau, M. Maglione, and G. Guegan, in European Microwave Integrated Circuits Conference (2013), pp. 460-463.

169. A. Ghalem, F. Ponchel, D. Remiens, J.F. Legier, and T. Lasri, IEEE Trans. Ultrason. Ferroelectr., 60, 880 (2013).

170. A. Khalfallaoui, G. Velu, L. Burgnies, and J.C. Carru, IEEE Trans. Ultrason. Ferroelectr., 57, 1029 (2010).

171. K. Nadaud, C. Borderon, S. Pavy, and H.W. Gundel, in IEEE International Symposium on the Applications of Ferroelectric and Workshop on the Piezoresponse Force Microscopy (2013), pp. 145-148.

172. H. Dong, D. Jin, C. Xie, J. Cheng, J. Chen, and J. Chen, in Joint IEEE International Symposium on the Applications of Ferroelectrics, International Workshop on Acoustic Transduction Materials and Devices and Workshop on Piezoresponse Force. Microscopy (2014), pp. 1-4.

173. L. Liu, L.B. Kong, W.Y. Yin, and S. Matitsine, IEEE Trans. Electromagn. Compon., 53, 943 (2011).

174. B. Zhao and C.B. Park, J. Mater. Chem. C, 5, 6954 (2017)

175. C. Morales, J. Dewdney, S. Pal, K. Stojak, H. Srikanth, J. Wang, and T. Weller, in IEEE MTT-S International Microwave Symposium Digest (2010), pp. 1340-1343.

176. S. De Blasi, P. Queffelec, S. Dubourg, O. Bodin, and M. Ledieu, IEEE Trans. Magn., 43, 2651(2007). 
177. J. Devkota, P. Colosimo, A. Chen, V. Larin, H. Srikanth, and M. Phan, J. Appl. Phys., 115, 17A525 (2014).

178. A. Tombak, J.P. Maria, F. Ayguavives, Z. Jin, G.T. Stauf, A.I. Kingon, and A. Mortazawi, IEEE Microw. Wirel. Compon, 12, 3 (2002).

179. M. Noda, Y. Sasaki, D. Popovici, M. Okuyama, and M. Komaru, in IEEE MTT-S International Microwave Symposium Digest (2005), pp. 1267-1270.

180. S. Bonetti, J.Y. Kim, S.I. Khartsev, and A.M. Grishin, in IEEE International Symposium on the Applications of Ferroelectrics (2006), pp. 344-347.

181. Y. Lee, Y. Hong, D. Kim, and K. Ko, Electron. Lett., 42, 851 (2006).

182. Y.H. Chun, C. Fragkiadakis, P. Bao, A. Luker, R.V. Wright, J.S. Hong, P.B. Kirby, Q. Zhang, T.J. Jackson, and M.J. Lancaster, in European Microwave Conference (2008), pp. $13-16$.

183. R. Follmann, D. Kother, M.A. Campo, T. Franke, A. Gabler, R. Jakoby, A. Manabe, T. Rabe, A. Heunisch, and A. Rauch, in IEEE-APS Topical Conference on Antennas and Propagation in Wireless. Communications (2013), pp. 9093.

184. Y. Malallah, D. Venkatesh, K. Alhassoon, A. Daryoush, C. Chinnasamy, M. Marinescu, and H. Gundel, in Mediterranean Microwave Symposium (MMS) (2016), pp. 1-4.

185. J. Park, J.W. Lu, D.S. Boesch, S. Stemmer, and R.A. York, IEEE Microw. Wirel. Compon., 16, 264 (2006).

186. M. Haghzadeh, C. Armiento, and A. Akyurtlu, in IEEE MTT-S International Microwave Symposium (2016), pp. 14.

187. T. Wang, Y. Peng, W. Jiang, Y.M. Huang, B.F. Rahman, R. Divan, D. Rosenmann, and G. Wang, IEEE Trans. Microw. Theory, 65, 504 (2017).

188. S. Bildik, C. Fritzsch, A. Moessinger, and R. Jakoby, in German Microwave Conference (2010), pp. 1-4.

189. M. Roig, M. Maasch, C. Damm, and R. Jakoby, in European Microwave Conference (2014), pp. 331-334.

190. G. Deng, T. Xia, S. Jing, J. Yang, H. Lu, and Z. Yin, IEEE Antennas Wirel. Propag., 16, 2062 (2017).

191. B.A. Belyaev, K.V. Lemberg, A.M. Serzhantov, A.A. Leksikov, Y.F. Bal'va, and A.A. Leksikov, IEEE Trans. Magn., 51, 1 (2015).

192. T. Zervos, D. Stamopoulos, F. Lazarakis, A. Alexandridis, M. Pissas, T. Giannakopoulou, and K. Dangakis, in European Conference on Antennas and Propagation (2009), pp. 1904-1908.

193. K. Bi, L. Zeng, K. Chai, Z. Fan, L. Liu, Q. Wang, and M. Lei, Appl. Phys. Lett., 107, 064103 (2015).

194. http://www.metamorphose-vi.org/index.php/metamaterials.

195. A. Sihvola, Metamaterials, 1, 2 (2007).

196. F. Bilotti and L. Sevgi, Int. J. RF. Microw. C. E., 22, 422 (2012).

197. J.C. Bose, Proc. R. Soc. Lond., 63, 146 (1898).

198. L. Mandel'shtam, Zh. Eksp. Teor. Fiz, 15, 475 (1945).

199. V.G. Veselago, Sov. Phys. Uspekhi, 10, 509 (1968).

200. J.B. Pendry, A.J. Holden, D.J. Robbins, and W. Stewart, IEEE Trans. Microw. Theory, 47, 2075 (1999).

201. R.A. Shelby, D.R. Smith, and S. Schultz, Science, 292, 77 (2001).

202. A. Alu, N. Engheta, A. Erentok, and R.W. Ziolkowski, IEEE Antennas Propag. Mag., 49, 23 (2007).

203. http://archive.li/wfwgr.

204. S.F. Mahmoud, IEEE Antennas Wirel. Propag., 3, 19 (2004).

205. T. Rowe, P. Forbes, J.H. Booske, and N. Behdad, IEEE Trans. Plasma Sci., 45, 654 (2017).

206. S. Lim, C. Caloz, and T. Itoh, IEEE Trans. Microw. Theory, 52,2678 (2004).

207. S. Wang, A. Feresidis, G. Goussetis, and J. Vardaxoglou, IEE Proc. Microw. Anten. Propag., 153, 1 (2006).

208. R.W. Ziolkowski and A. Erentok, IEEE Trans. Antennas Propag., 54, 2113 (2006)

209. D. Laila, S. Raman, S.M. Nair, C.K. Aanandan, K Vasudevan, and P. Mohanan, Prog. Electromagn. Res., 17, 39 (2010).
210. S.V. Pushpakaran, R.K. Raj, A. Pradeep, L. Ouseph, M. Hari, A. Chandroth, M. Pezholil, and V. Kesavath, Appl. Phys. Lett., 104, 064102 (2014).

211. M. Jiang, Z.N. Chen, Y. Zhang, W. Hong, and X. Xuan, IEEE Trans. Antennas Propag., 65, 464 (2017).

212. K. Buell, H. Mosallaei, and K. Sarabandi, in IEEE Antennas and Propagation Society International Symposium (2005), pp. 574-577.

213. A. Alu, F. Bilotti, N. Engheta, and L. Vegni, IEEE Trans. Antennas Propag., 54, 1632 (2006).

214. S.B. Yeap, X. Qing, Z.N. Chen, and M. Sun, in International Workshop on Antenna Technology (iWAT) (2015), pp. 202-205.

215. D. Chaurasiya, S. Ghosh, S. Bhattacharyya, and K.V. Srivastava, in IEEE International Microwave and $R F$ Conference (2014), pp. 96-99.

216. P. Munaga, S. Ghosh, S. Bhattacharyya, D. Chaurasiya, and K.V. Srivastava, in European Conference on Antennas and Propagation (2015), pp. 1-4.

217. S. Bobrovskii, I. Iakubov, A. Lagarkov, A. Osipov, and K. Rozanov, IEEE Trans. Magn., 53, 1 (2017).

218. P. Anju, S. Mridula, and P. Mohanan, Int. J. Commun. Antennas Propag., 3, 181 (2013).

219. Y. Yakhlef, M. Benhabiles, L. Benkhaoua, and M. Riabi, in IEEE MTT-S International Microwave Bio Conference (2017), pp. 1-4.

220. V. Sarin, M. Jayakrishnan, P. Vinesh, C. Aanandan, P. Mohanan, and K. Vasudevan, Can. J. Phys., 95, 927 (2017).

221. I. Martinez, A.H. Panaretos, D.H. Werner, IEEE Antennas Wirel. Propag., 16, 1915 (2017).

222. Y. Fan, H.C. Zhang, J.Y. Yin, L. Xu, D.S. Nagarkoti, Y. Hao, and T.J. Cui, IEEE Antennas Wirel. Propag., 15, 1913 (2016).

223. Y.H. Hu, H.F. Zhang, L.L. Wang, W. Shi, and C.Y. Mao, in Progress in Electromagnetic Research Symposium (PIERS) (2016), pp. 1916-1918.

224. C.L. Holloway, E.F. Kuester, J.A. Gordon, J. O'Hara, J. Booth, and D.R. Smith, IEEE Antennas Propag. Mag., 54, 10 (2012).

225. A.K. Neha, in International Conference on Next Generation Computing Technologies (2016), pp. 197-201.

226. B. Dong, W. Zhu, Y. Fu, J. Tsai, H. Cai, D. Kwong, E. Li, E. Rius, and A. Liu, in International Solid-State Sensors. Actuators and Microsystems Conference (2011), pp. 530 533.

227. J.A. Gordon, C.L. Holloway, J. Booth, S. Kim, Y. Wang, J. Baker-Jarvis, and D.R. Novotny, Phys. Rev. B, 83, 205130 (2011).

228. A. Ali and Z. Hu, IEEE Antennas Wirel. Propag., 7, 210 (2008).

229. H. Ouchi and S. Kawashima, Jpn. J. Appl. Phys., 24, 60 (1985).

230. M. Sebastian, R. Ubic, and H. Jantunen, Int. Mater. Rev. 60, $392(2015)$.

231. A. Wypych-Puszkarz, I. Bobowska, A.Wrzesinska, A. Opasinska, W. Maniukiewicz, P. Wojciechowski, and J. Ulanski, J. Nanomater., 2017, 9 (2017).

232. K. Surendran, N. Santha, P. Mohanan, and M. Sebastian, Eur. Phys. J. B., 41, 301 (2004).

233. D. Thomas and M.T. Sebastian, J. Am. Ceram. Soc., 93, 3828 (2010).

234. S.O. Yoon, S.H. Shim, K.S. Kim, J.G. Park, and S. Kim, Ceram. Int., 35, 1271 (2009).

235. H. Zhou, F. He, X. Chen, J. Chen, and L. Fang, J. Mater. Sci.- Mater. Electron., 25, 1480 (2014).

236. R. Richtmyer, J. Appl. Phys., 10, 391 (1939).

237. M. Abedian, S.K.A. Rahim, C. Fumeaux, S. Danesh, Y.C. Lo, and M.H. Jamaluddin, IET Microw. Antenna Propag., $11,1524(2017)$

238. J.X. Chen, J. Li, Y. Zhan, W. Qin, J. Shi, and Z.H. Bao, IEEE Trans. Microw. Theory, 65, 1226 (2017).

239. L. Zhou, W.Y. Yin, J. Wang, and L.S. Wu, IEEE Trans. Compon. Packag. Manuf., 3, 1008 (2013). 
240. S. Maity and B. Gupta, AEU-Int. J. Electron. Commun., 83, 451 (2018).

241. D. Guha and C. Kumar, IEEE Antennas Propag. Mag., 58, 45 (2016).

242. M. Mrnka, M. Cupal, Z. Raida, A. Pietrikova, and D. Kocur, IET Microw. Antenna Propag., 12, 662 (2018).

243. R. Chakraborty, M. Pal, and R. Ghatak, AEU-Int. J. Electron. Commun., 83, 348 (2018).

244. A. Petosa, A. Ittipiboon, Y. Antar, D. Roscoe, and M. Cuhaci, IEEE Antennas Propag. Mag., 40, 35 (1998).

245. Y.M. Antar, IET Circuits Device. Syst., 2, 133 (2008).

246. A. Petosa and A. Ittipiboon, IEEE Antennas Propag. Mag., 52,91 (2010).

247. R. Cameron, C. Kudsia, and R. Mansour, Microwave Filters for Communication Systems: Fundamentals, Design, and Applications (Wiley, Hoboken, 2018), pp. 517-543.

248. C. Wang and K.A. Zaki, IEEE Microw. Mag., 8, 115 (2007).

249. M. Sebastian, S. George, P. Anjana, S. Thomas, and G. Subodh, in International Conference on Recent Advances in Microwave Theory and Applications (2008), p. 161.

250. G. Subodh, V. Deepu, P. Mohanan, and M. Sebastian, Appl. Phys. Lett., 95, 062903 (2009).

251. G. Subodh, V. Deepu, P. Mohanan, and M. Sebastian, Polym. Eng. Sci., 49, 1218 (2009).

252. S. Koulouridis, G. Kiziltas, Y. Zhou, D.J. Hansford, and J.L. Volakis, IEEE Trans. Microw. Theory, 54, 4202 (2006).
253. P.S. Anjana, M.T. Sebastian, M.N. Suma, and P. Mohanan, Int. J. Appl. Ceram. Technol., 5, 325 (2008).

254. S. Thomas, S. Raman, P. Mohanan, and M. Sebastian, Compos. A Appl. Sci., 41, 1148 (2010).

255. T. Sasikala, S. Raman, P. Mohanan, C. Pavithran, and M. Sebastian, J. Polym. Res., 18, 811 (2011).

256. C.L. Lo, J.G. Duh, B.S. Chiou, and W.H. Lee, J. Am. Ceram. Soc., 85, 2230 (2002).

257. H. Braun, M. Hovhannisyan, Y. Zheng, A. Mehmood, R. Jakoby, O. Leisten, and M. Letz, in European Microwave Conference (2013), pp. 1167-1170.

258. S. George, M.T. Sebastian, S. Raman, and P. Mohanan, Int. J. Appl. Ceram. Technol., 8, 172 (2011).

259. A. Vahdati, A. Lamminen, M. Varonen, J. Saily, M. Lahti, K. Kautio, M. Lahdes, D. Parveg, D. Karaca, and K.A. Halonen, EEE Trans. Antennas Propag., 65, 6363 (2017).

260. D. Titz, R. Pilard, F. Gianesello, F. Ferrero, C. Luxey, P. Brachat, G. Jacquemod, and D. Gloria, IEEE Antennas Wirel. Propag., 11, 576 (2012).

261. Z. Di, P. Li-Xia, Q. Ze-Ming, J. Biao-Bing, and Y. Xi, Sci. 4, 5980 (2014).

Publisher's Note Springer Nature remains neutral with regard to jurisdictional claims in published maps and institutional affiliations 